\title{
Estudio comparativo de la composición química y la actividad antioxidante de los aceites esenciales de algunas plantas del género Lippia (Verbenaceae) cultivadas en Colombia.
}

\author{
Elena E. Stashenko*, Jairo René Martínez, Diego Camilo Durán, Yuri Córdoba, Deyanira Caballero
}

CIBIMOL-CENIVAM, Facultad de Ciencias, Universidad Industrial de Santander, Bucaramanga, Colombia

\begin{abstract}
Resumen
Se presentan varios resultados obtenidos en investigaciones de aceites esenciales de especies vegetales aromáticas que crecen en Colombia, se destaca la necesidad de investigar una gran variedad de tópicos que permitan brindar el soporte científico para el desarrollo de la agroindustria de aceites esenciales, y se presentan en detalle resultados de la caracterización química y la evaluación de la capacidad antioxidante de 12 aceites esenciales de 7 especies del género Lippia. La determinación de la composición química de 12 aceites esenciales de plantas del género Lippia, por cromatografía de gases acoplada a espectrometría de masas, mostró que los constituyentes más abundantes y más frecuentes son sustancias oxigenadas. La diversidad composicional se manifiesta en la isomería óptica, en la existencia de quimiotipos con pocas sustancias en común, y en los diversos modos de atrapamiento de radicales, según lo evidenciaron los resultados de las evaluaciones de capacidad antioxidante por los métodos ORAC y TEAC. Mientras que el limoneno y el linalol se encontraron en todos los aceites, 19 de los 102 constituyentes identificados en el conjunto de aceites se hallaron en solo una ocasión. Los aceites esenciales de las especies del género Lippia estudiados mostraron una capacidad antioxidante mayor que la exhibida por el $\alpha$-tocoferol o el BHT, que son utilizados comúnmente como antioxidantes en productos comerciales de consumo humano. Varias especies tuvieron un rendimiento de extracción relativamente alto $(1,5-4,4 \%)$, que junto con la alta capacidad antioxidante registrada, convierte a sus aceites esenciales en buenos candidatos para sustituir a antioxidantes sintéticos.
\end{abstract}

Palabras clave: Lippia alba; Lippia origanoides; quimiotipo; carvona; timol; carvacrol; ORAC

Comparative study of chemical composition and antioxidant activity of essential oils of some plants from genus Lippia (Verbenaceae) grown in Colombia.

\begin{abstract}
Various results obtained from research on essential oils of vegetal aromatic species growing in Colombia are presented. In order to offer good scientific support to the essential oils agro-industry, the need for investigating a wide variety of topics is emphasized. Results of the chemical characterization and the antioxidant activity evaluation of 12 essential oils of 7 Lippia species are presented. The determination of the chemical composition of 12 essential oils of plants from genus Lippia, using gas chromatography coupled to mass spectrometry, showed that the most abundant and frequent constituents are oxygenated substances. The compositional diversity is manifested in optical isomers, in the existence of chemotypes with few substances in common, and in the diverse radical trapping modes, as evidenced by the ORAC and TEAC antioxidant capacity evaluations. While limonene and linalool were found in all the oils, 19 out of the 102 identified constituents of the set of oils studied were found in only one instance. The studied genus Lippia essential oils showed an antioxidant capacity higher than that exhibited by $\alpha$-tocopherol or BHT, which are commonly used as antioxidants in commercial products for human consumption. Several species had a relatively high $(1,5-4,4 \%)$ essential oil extraction yield, which together with their high antioxidant capacity convert these oils into good candidates to replace synthetic antioxidants.
\end{abstract}

Key words: Lippia alba; Lippia origanoides; chemotype; carvone; thymol; carvacrol; ORAC

\section{Introducción}

En Colombia, la mayoría de los cultivos industriales, provienen de especies vegetales introducidas, e.g., café, caña de azúcar, sorgo, arroz, trigo, palma africana, cítricos, entre otros. A pesar de que los pueblos nativos utilizaban -tanto con propósitos culinarios, como curativos y mágico-religiosos-, una gran variedad de plantas, frutos, raíces y tubérculos endógenos, propios de Sur América, y que los productos de interés agro-económico y alimenticio tan importantes como papa, tomate, maíz, tabaco, batata, marañón, entre otros, fueron "transferidos" al resto del mundo, la gran mayoría de plantas medicinales y, sobre todo, aromáticas, conocidas 
por el común de la gente hoy en día, son exógenas, i.e., han sido -a lo largo de más de quinientos años-, introducidas a Colombia. Romero y albahacas, tomillo y mentas, anís [1], orégano [2], mejorana, ruda [3, 4], provienen del Mediterráneo; limonaria y citronela [5], jengibre, cítricos [68], vetiver, ylang-ylang [9-11], patchoulí (pachulí) llegaron de Asia, geranio, del sur de África; pero, hoy en día, estas son plantas comunes en Colombia, asilvestradas o cultivadas, que se utilizan ampliamente con diferentes propósitos, tanto en medicina popular, como en culinaria.

Las plantas aromáticas nativas, sin embargo, han sido estudiadas menos, aunque merecen mucho mayor atención, no sólo porque algunas son escasas o en peligro de extinción, sino porque su potencial económico es alto, tanto como fuente -poco explorada-, de nuevos compuestos bioactivos, como también en calidad de materia prima y como cultivos promisorios para obtener aceites esenciales, extractos $\mathrm{u}$ oleorresinas, con características y aplicaciones nuevas [12-16]. El Programa Nacional para el Desarrollo de la Biotecnología y la Agroindustria en Colombia (Colciencias), CONPES 3582 y 3697, la Cadena de Plantas Aromáticas, Medicinales, Condimentarias y Afines del Ministerio de Agricultura y Desarrollo Rural, incentivan los estudios académicos y agro-industriales sobre plantas aromáticas nativas $[12,17-24]$, con miras a desarrollar sus cultivos tecnificados y obtener aceites esenciales. Esto permitiría crear nuevas fuentes de trabajo en el campo y generaría una oportunidad agrícola viable para la sustitución de cultivos de uso ilícito. Teniendo cultivos tecnificados -preferiblemente orgánicos-, de plantas aromáticas, que son de rápido crecimiento, se podría desarrollar una nueva industria para la obtención de sub-productos agrícolas, e.g., aceites esenciales, extractos, derivados con un alto valor agregado. Esto no solamente generaría empleo, sino permitiría reemplazar muchos ingredientes naturales y sintéticos, que, en Colombia, actualmente se importan en su totalidad; a la vez, contribuiría al desarrollo de un sector agrícola moderno e innovador.

El desarrollo de la industria de aceites esenciales implica estudios no solamente a nivel agronómico y sobre la factibilidad económica y de mercados, sino, paralelamente, una investigación detallada de la composición química, propiedades físico-químicas (ficha técnica) y biológicas de los aceites esenciales obtenidos de las plantas cultivadas, introducidas o nativas. En el Centro Nacional de Investigaciones para la Agroindustrialización de Especies Vegetales, Aromáticas y Medicinales Tropicales, CENIVAM, se han estudiado sistemáticamente varias especies vegetales

\footnotetext{
*Correspondencia:

Elena E. Stashenko, elena@tucan.uis.edu.co

Recibido: 11 de marzo de 2013

Aceptado: 31 de marzo de 2014
}

aromáticas tanto exógenas [1-11], como nativas, entre estas, Copaifera officinalis [17], Spilanthes americana [18], Lepechinia schiediana [19], Lippia alba [20], Xylopia aromatica [21], Hyptis umbrosa [22], Callistemon speciosus [23] y Lippa origanoides [24]. En estos estudios, se ha hecho el énfasis muy especial en la comparación de métodos de extracción de metabolitos secundarios volátiles [3, 11, 17$21,25,26]$, en el análisis de aceites esenciales y fracciones volátiles por cromatografía de gases (GC) con diferentes sistemas de detección $[4,7,18]$; así mismo, en el estudio de la transformación catalítica de aceites $[1,8,17]$ y en la investigación de la actividad biológica de extractos y aceites obtenidos [2, 19, 20, 23, 24, 27, 28].

Las especies vegetales aromáticas de la familia Verbenaceae figuran dentro de las plantas aromáticas, que se estudian, de manera sistemática, en el CENIVAM. Las Verbenáceas incluyen más de 30 géneros y alrededor de 1200 especies herbáceas, arbustos o árboles, distribuidas principalmente en zonas tropicales. Se destacan los géneros Aloysia, Duranta, Lantana, Lippia, Petrea, Phyla, Verbena, entre otros. Muchas plantas de esta familia poseen flores vistosas y de colores alegres.

Dentro de la familia Verbenaceae, el género Lippia llama mucho la atención de los investigadores, por la variedad de las especies que posee, diferentes quimiotipos, y sus diversos usos en medicina y culinaria [29]. Las propiedades organolépticas atractivas y actividades biológicas, e.g., antifúngicas y antibacterianas, de los aceites esenciales de plantas del género Lippia, las convierten en buenos candidatos como ingredientes naturales en productos cosméticos y de aseo personal. Se observa una gran diversidad composicional en los aceites esenciales y extractos, obtenidos de Lippias; éstos poseen, además, muy variada actividad biológica [30-38].

\subsection{Especies vegetales del género Lippia.}

El género Lippia Houst. pertenece a la familia Verbenaceae Juss., y comprende alrededor de 200 especies, entre hierbas, arbustos y pequeños árboles. La palabra Lippia se derivó del nombre de Auguste Lippi, botánico italiano nacido en París en 1678 [39]. Las plantas de Lippia spp. se encuentran distribuidas por toda América del Sur, América Central y África Tropical [40]. La mayoría de las especies de Lippia son plantas aromáticas, con un olor penetrante; muchas de ellas, por esta propiedad, se emplean en culinaria y, por sus propiedades curativas, en medicina popular. En la Figura 1 se presentan diversas plantas aromáticas del género Lippia; algunas, durante los últimos 15 años, han sido objeto de investigación del CENIVAM.

El estudio cienciométrico realizado sobre las plantas del género Lippia muestra que atraen la atención de investigadores en distintas países del mundo. En la base de datos de Scopus 
A. Lippia origanoides

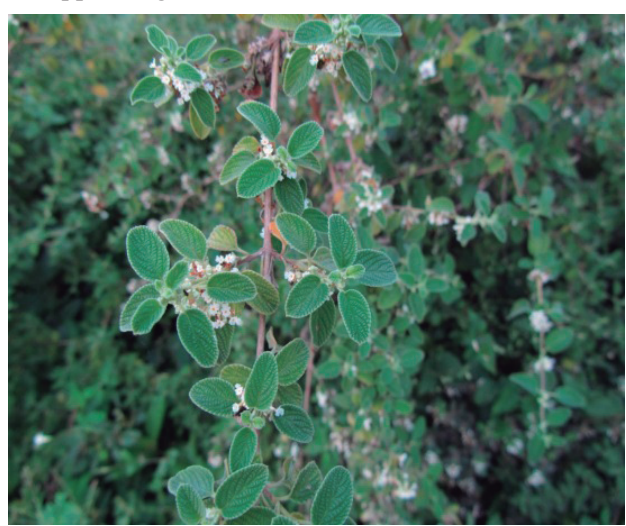

C. Lippia micromera

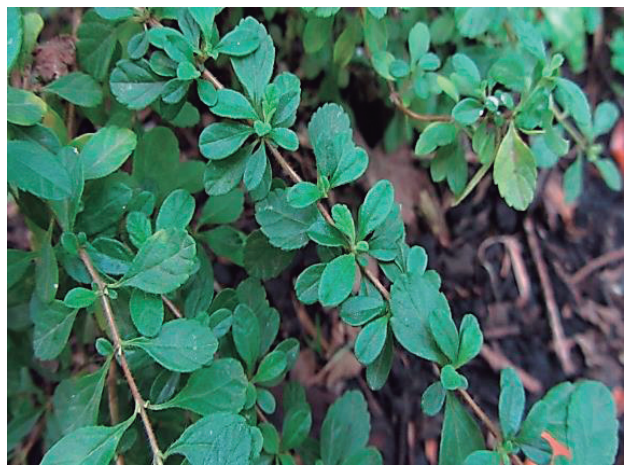

E. Lippia alba

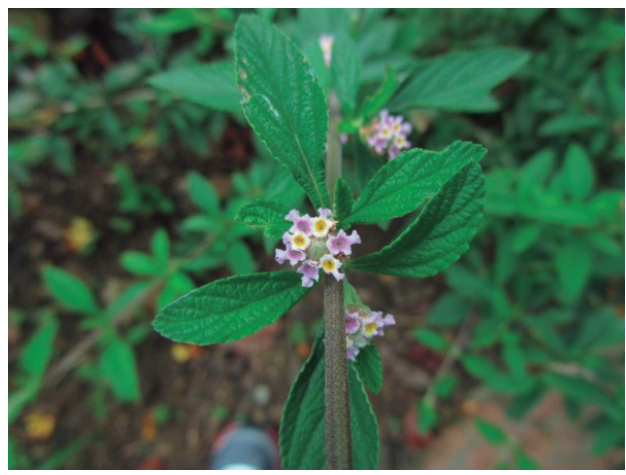

\section{B. Lippia americana}

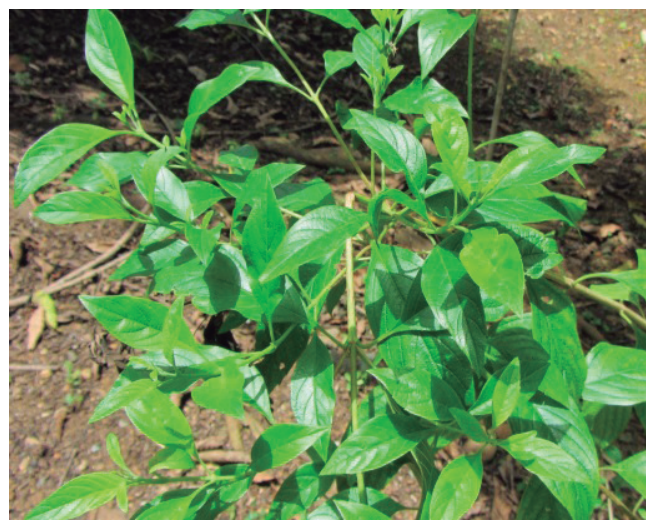

D. Lippia dulcis

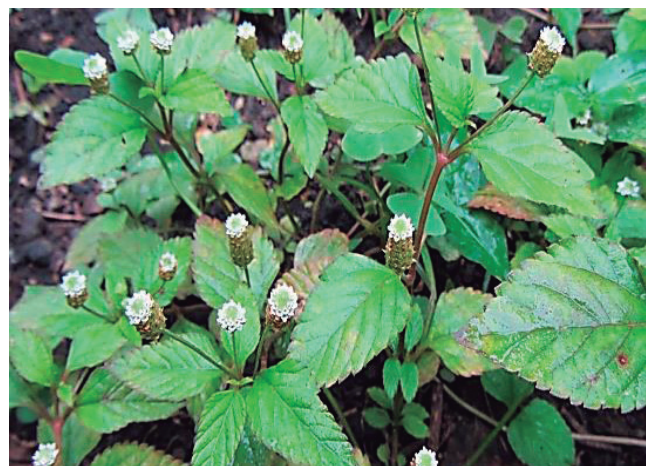

F. Lippia graveolens

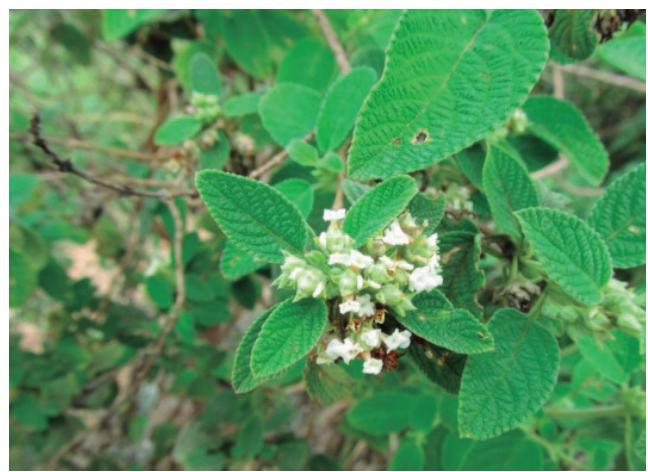

Figura 1. A, Planta de orégano de monte (Lippia origanoides, Familia Verbenácea) en florecimiento. Arbusto pequeño, leñoso, aromático, de crecimiento relativamente rápido. En Colombia, se han encontrado tres quimiotipos, ricos en timol, carvacrol o en $\alpha$ - y b- felandrenos. B,. Planta de Lippia americana (Familia Verbenácea). Arbusto poco aromático, de crecimiento rápido, contiene aceite esencial, rico en monoterpenos (sabineno, felandrenos, limoneno, terpinenos) y sesquiterpenos (cariofileno, germacrenos). C, Planta de Lippia micromera (Familia Verbenácea), a menudo, llamada "falso orégano"; pequeño arbusto, muy aromático, de crecimiento lento. El aceite esencial contiene sabineno, limoneno, p-cimeno, terpineno, timol y sus éteres. D, Planta de Lippia dulcis (Familia Verbenácea) en florecimiento, llamada, a menudo, "Lippia mexicana" o "Hierba dulce de Aztecas"; proviene de México y América Central, aromática y de rápido crecimiento. Contiene la hernandulcina, un sesquiterpenoide, mil veces más dulce que la sacarosa, pero inestable térmicamente. E, Planta de pronto alivio (Lippia alba, Familia Verbenácea) en florecimiento. Pequeño arbusto, muy aromático, de rápido crecimiento, posee varios quimiotipos; en Colombia, se han encontrado los quimiotipos, ricos en carvona, citral y un quimiotipo híbrido (carvona + citral). Otro quimiotipo de Lippia alba, i.e., rico en ocimenonas (tagetenonas), posiblemente, fue introducido a Colombia, proveniente de Centroamérica o Argentina. F, Planta de orégano mexicano (Lippia graveolens, Familia Verbenácea) en florecimiento. Arbusto muy aromático, de rápido crecimiento, su aceite esencial, rico en timol y carvacrol y sus éteres, posee propiedades antifúngicas y antibacterianas. 
(Elsevier) aparecen 746 registros correspondientes a los años 1980-2012, sobre las especies del género Lippia. La mayoría de artículos científicos sobre Lippias han sido publicados en las siguientes revistas científicas: Journal of Ethnopharmacology, Journal of Essential Oil Research, Revista Brasileira de Plantas Medicinales, Flavour and Fragrance Journal, Journal of Natural Products, entre otras. Los estudios sobre Lippias corresponden a diferentes áreas de conocimiento, pero, fundamentalmente, se desarrollan en los campos de Agricultura y Ciencias Biológicas, Farmacología, Toxicología y Ciencias Farmacéuticas, Química, Medicina, Bioquímica, Genética y Biología Molecular. En la Figura 2 se observa la tendencia muy marcada en el crecimiento del número de estudios sobre las especies Lippia spp., sus metabolitos secundarios y la actividad biológica: en los últimos 10 años, este aumento es más notorio.

Brasil, Argentina, México, EE.UU. y Suráfrica figuran entre los países donde se ha realizado el mayor número de estudios (publicados) sobre las plantas del género Lippia (Figura 3). Entre los países latinoamericanos, Colombia ocupa el puesto cuarto, con 31 artículos, después de Brasil (245), Argentina (72) y México (56) (Figura 4).

Los aceites esenciales, aislados de las especies del género Lippia, se destacan no solamente por la variedad química de sus componentes, sino por su diversa actividad biológica. Aceites esenciales y extractos, obtenidos de Lippias, poseen propiedades antiinflamatorias, antibacterianas, antifúngicas, antivirales y antioxidantes; la última actividad, quizás, es de las más estudiadas. En la Tabla 1 aparece la información del número de publicaciones sobre distintas actividades biológicas de algunas plantas de Lippia spp. más estudiadas.
En la Tabla 2 se resume la información sobre la composición química (componentes principales) y la actividad biológica más estudiada de cinco especies vegetales del género Lippia, algunas de las cuales han sido también el objeto de nuestro continuo estudio.

\subsection{Análisis de los aceites esenciales}

El análisis de los aceites esenciales comprende el estudio de tres aspectos: 1. Análisis sensorial, realizado por "narices" o panel de expertos entrenados en evaluar las características organolépticas del producto, apreciar su buqué, sus notas odoríferas placenteras u off-flavour. 2. Propiedades físicoquímicas, que son pruebas estandarizadas [15], que miden características físicas del aceite, e.g., su densidad, contenido de humedad, índice de refracción, puntos de ignición y congelación, etc. y 3. Composición química, que se determina por cromatografía de gases (GC) con sistemas de detección de ionización en llama, GC-FID (FID, Flame Ionization Detector, por sus siglas en inglés) y espectrometría de masas GC-MS (MS, Mass Spectrometry, por sus siglas en inglés). El análisis GC-FID se emplea para hacer la cuantificación de los componentes presentes en el aceite y el análisis GC-MS, para su identificación.

El análisis cromatográfico de los aceites esenciales, que pueden contener más de 200 componentes, entre ellos, muchos isómeros, se realiza en columnas capilares largas, de 50 o 60 $\mathrm{m}$, con fases estacionarias apolar [poli (dimetilsiloxano) o 5\%-fenil-poli(dimetilsiloxano)] y polar [poli(etilenglicol), Carbowax)]; se calculan los índices de retención lineales (LRI) en ambas columnas y se obtienen los espectros de masas por impacto de electrones (EI) con energía de $70 \mathrm{eV}$.

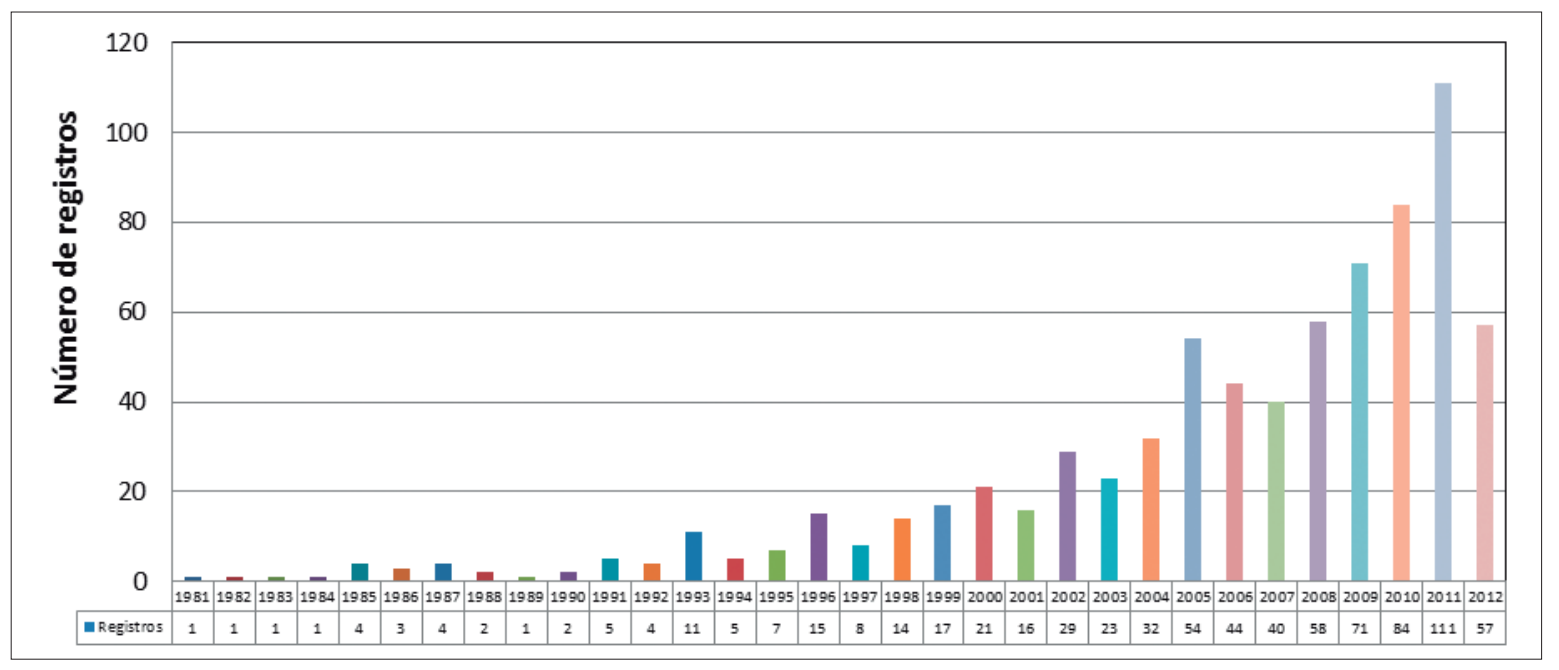

Figura 2. Número de publicaciones científicas, por año, sobre las especies del género Lippia. Período de observación: 1980-2012. Fecha de consulta: 27 de septiembre de 2012. Ecuación de búsqueda: TITLE-ABS-KEY (“Lippia” OR “Lippia sp”) AND DOCTYPE (ar) AND PUBYEAR > 1979. Base de datos: Scopus (Elsevier). 


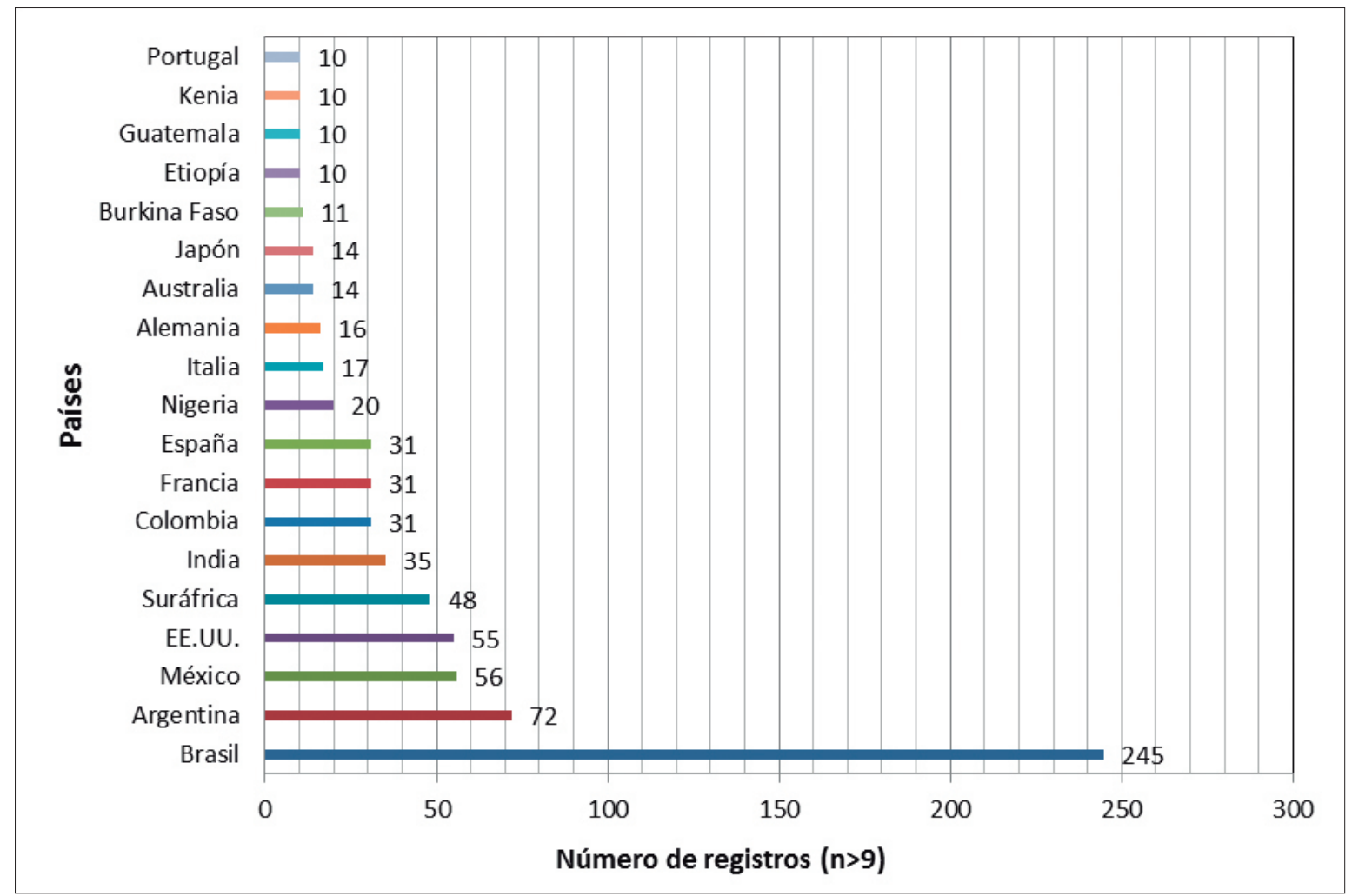

Figura 3. Número de publicaciones científicas, por país, sobre las especies del género Lippia. Período de observación: 1980-2012. Fecha de consulta: 27 de septiembre de 2012. Ecuación de búsqueda: TITLE-ABS-KEY (“Lippia” OR “Lippia sp”) AND DOCTYPE (ar) AND PUBYEAR > 1979. Base de datos: Scopus (Elsevier).

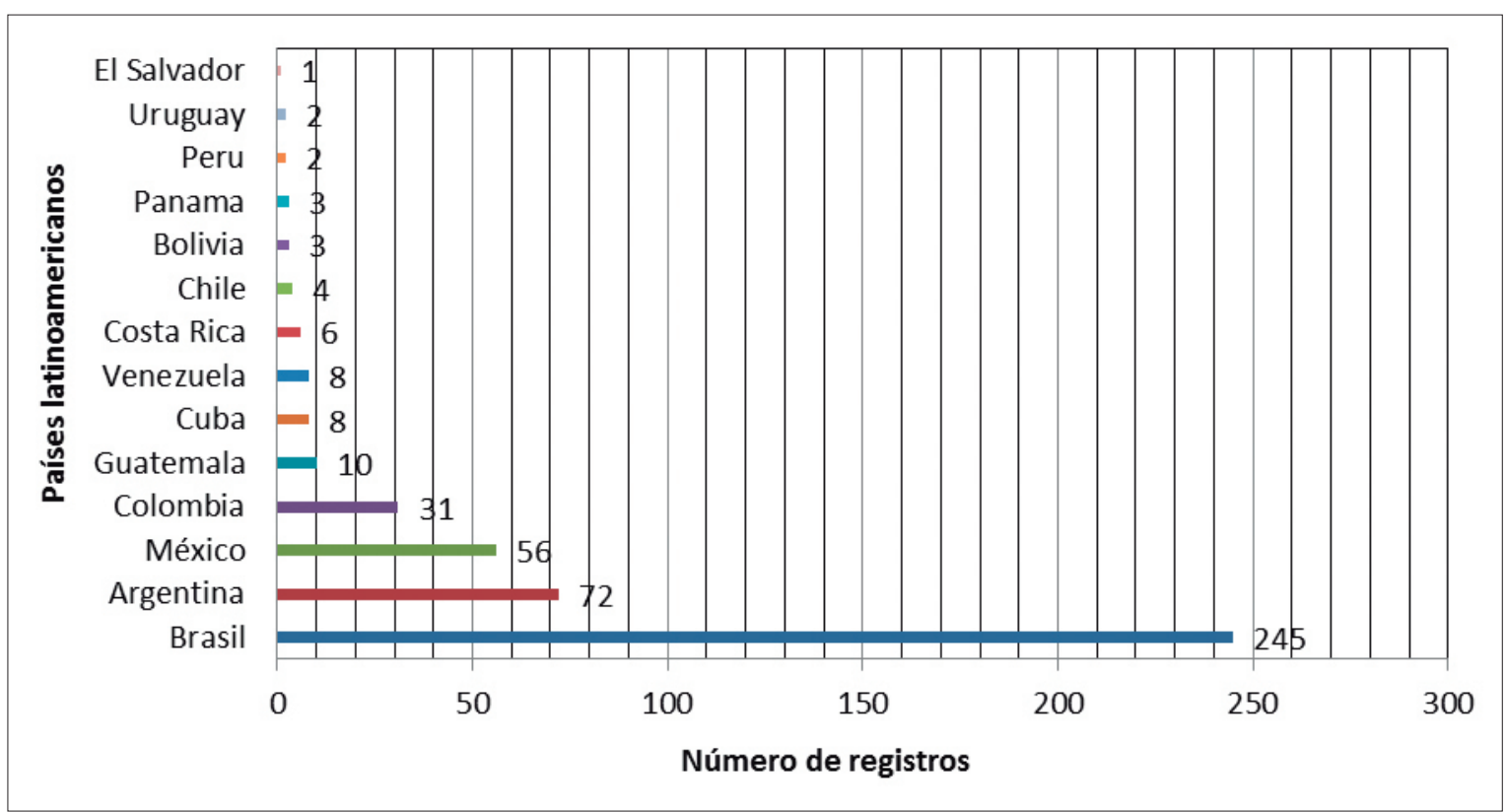

Figura 4. Número de publicaciones científicas, realizadas en los países latinoamericanos, sobre las especies del género Lippia. Período de observación: 1980-2012. Fecha de consulta: 27 de septiembre de 2012. Ecuación de búsqueda: TITLE-ABS-KEY ("Lippia” OR "Lippia sp") AND DOCTYPE (ar) AND PUBYEAR > 1979. Base de datos: Scopus (Elsevier). 
Para la identificación de componentes en los aceites esenciales se utilizan sus tiempos e índices de retención y espectros de masas, se emplean bases de datos de espectros de masas (Adams, Wiley y NIST) y, para la identificación confirmatoria y la cuantificación, se requiere el uso de sustancias-patrón [41-49].
La composición química detallada de los aceites esenciales es muy importante para su evaluación sensorial, correlación con propiedades biológicas, detección de adulteraciones y determinación de origen, análisis de trazas, establecimiento del precio del producto y su aceptación en el mercado, entre otros aspectos. Por ejemplo, se han registrado -a través

Tabla 1. Número de publicaciones científicas relacionadas con el estudio de la actividad biológica de algunas plantas más estudiadas del género Lippia. Período de observación: 1980-2012. Fecha de consulta: 27 de septiembre de 2012. Ecuación de búsqueda: TITLE-ABS-KEY ("Lippia” OR “Lippia sp") AND DOCTYPE (ar) AND PUBYEAR > 1979. Base de datos: Scopus (Elsevier).

\begin{tabular}{|c|c|c|c|c|c|c|c|c|c|c|c|c|}
\hline $\begin{array}{l}\text { Actividad } \\
\text { biológica }\end{array}$ & $\begin{array}{c}\text { Lippia } \\
\text { alba }\end{array}$ & $\begin{array}{c}\text { Lippia } \\
\text { sidoides }\end{array}$ & $\begin{array}{c}\text { Lippia } \\
\text { multiflora }\end{array}$ & $\begin{array}{c}\text { Lippia } \\
\text { graveolens }\end{array}$ & $\begin{array}{c}\text { Lippia } \\
\text { javanica }\end{array}$ & $\begin{array}{c}\text { Lippia } \\
\text { turbinata }\end{array}$ & $\begin{array}{c}\text { Lippia } \\
\text { citriodora }\end{array}$ & $\begin{array}{c}\text { Lippia } \\
\text { nodiflora }\end{array}$ & $\begin{array}{c}\text { Lippia } \\
\text { gracilis }\end{array}$ & $\begin{array}{c}\text { Lippia } \\
\text { origanoides }\end{array}$ & $\begin{array}{c}\text { Lippia } \\
\text { beriandieri }\end{array}$ & $\begin{array}{c}\text { Lippia } \\
\text { chevalicri }\end{array}$ \\
\hline Antiinflamatoria & 4 & 3 & 3 & - & 2 & - & 2 & 3 & 3 & & & \\
\hline Antiviral & 6 & - & - & - & - & - & - & - & - & 1 & - & - \\
\hline Antioxidante & 9 & 3 & 5 & 6 & 4 & 2 & 7 & 6 & - & - & 2 & - \\
\hline Antimicrobiana & 10 & 10 & 5 & 12 & 8 & 4 & 3 & 3 & 3 & 2 & 2 & 3 \\
\hline Antifúngica & 10 & 7 & - & 11 & 4 & 2 & 1 & 2 & - & - & 2 & - \\
\hline Antibacteriana & 11 & 7 & 3 & 6 & 7 & 3 & 5 & 2 & 2 & - & - & 4 \\
\hline Antimalárica & - & - & 6 & - & 3 & - & - & - & - & - & - & 2 \\
\hline
\end{tabular}

Tabla 2. Estudio de la composición química y actividad biológica de las plantas de L. sidoides, L. origanoides, L.graveolens, L.citriodora y L.alba. Período de observación: 1980-2012. Fecha de consulta: 27 de septiembre de 2012. Ecuación de búsqueda: TITLE-ABS-KEY (“Lippia” OR "Lippia sp") AND DOCTYPE (ar) AND PUBYEAR > 1979. Base de datos: Scopus (Elsevier).

\begin{tabular}{|c|c|c|c|c|}
\hline \multirow[b]{2}{*}{ Especie vegetal } & \multirow{2}{*}{$\begin{array}{l}\mathrm{N}^{\circ} \text { total } \\
\text { de registros } \\
(1980-2012)\end{array}$} & \multicolumn{2}{|c|}{$\mathrm{N}^{\circ}$ de registros } & \multirow[b]{2}{*}{ Componentes mayoritarios del aceite esencial } \\
\hline & & $\begin{array}{c}\text { Países con mayor } \\
\mathbf{N}^{\circ} \text { de publicaciones }\end{array}$ & $\begin{array}{l}\text { Actividad biológica } \\
\text { estudiada }\end{array}$ & \\
\hline Lippia sidoides & 83 & $\begin{array}{l}\text { Brasil (79) } \\
\text { Italia (2) } \\
\text { Corea del Sur (2) } \\
\text { EE.UU. (2) }\end{array}$ & $\begin{array}{l}\text { Antimicrobiana (10) } \\
\text { Antifúngica (10) } \\
\text { Antibacteriana (7) } \\
\text { Insecticida (3) } \\
\text { Antioxidante (3) }\end{array}$ & $\begin{array}{l}\text { Felandrenos, } p \text {-cimeno, terpinenos, carvacrol, } \\
\text { timol, } \beta \text {-cariofileno }\end{array}$ \\
\hline Lippia origanoides & 22 & $\begin{array}{l}\text { Colombia (13) } \\
\text { Venezuela (6) } \\
\text { Brasil (3) } \\
\text { EE.UU. (1) }\end{array}$ & $\begin{array}{l}\text { Citotóxica (3) } \\
\text { Antimicrobiana (2) } \\
\text { Antifúngica (2) } \\
\text { Antigenotóxica (1) } \\
\text { Antioxidante (2) }\end{array}$ & $\begin{array}{l}\text { 1,8-Cineol, timol, carvacrol, ésteres y éteres } \\
\text { de timilo y carvacrilo, terpinenos, } p \text {-cimeno, } \\
\text { felandrenos, } \beta \text {-cariofileno }\end{array}$ \\
\hline Lippia graveolens & 46 & $\begin{array}{l}\text { México (30) } \\
\text { Brasil (7) } \\
\text { EE.UU. (5) } \\
\text { Guatemala (4) }\end{array}$ & $\begin{array}{l}\text { Antimicrobiana (12) } \\
\text { Antifúngica (11) } \\
\text { Antibacteriana (6) } \\
\text { Antioxidante (6) } \\
\text { Antiprotozoica (2) }\end{array}$ & $\begin{array}{l}\text { 1,8-Cineol, felandrenos, } p \text {-cimeno, terpinenos, } \\
\text { carvacrol, timol y sus éteres y ésteres, } \\
\beta \text {-cariofileno }\end{array}$ \\
\hline Lippia citriodora & 47 & $\begin{array}{l}\text { España (11) } \\
\text { Italia (5) } \\
\text { Francia (4) } \\
\text { Irán (4) }\end{array}$ & $\begin{array}{l}\text { Antioxidante (7) } \\
\text { Antibacteriana (5) } \\
\text { Antimicrobiana (3) } \\
\text { Antiinflamatoria (2) } \\
\text { Antifúngica (1) }\end{array}$ & $\begin{array}{l}\text { Limoneno, 1,8-cineol, geranial, neral, geraniol, } \\
\text { nerol y sus acetatos, isómeros de fotocitral, } \\
\text { carvacrol, } \alpha \text {-curcumeno }\end{array}$ \\
\hline Lippia alba & 163 & $\begin{array}{l}\text { Brasil (100) } \\
\text { Colombia (19) } \\
\text { India (14) } \\
\text { Argentina (6) }\end{array}$ & $\begin{array}{l}\text { Antibacteriana (11) } \\
\text { Antifúngica (10) } \\
\text { Citotóxica (10) } \\
\text { Antimicrobiana (10) } \\
\text { Antioxidante (9) }\end{array}$ & $\begin{array}{l}\text { Dependen del quimiotipo: } \\
\text { Limoneno, } p \text {-cimeno, carvona, piperitona, } \\
\text { piperitenona, geranial, neral, geraniol, nerol, } \\
\text { linalool, } \beta \text {-cariofileno y su óxido, germacreno D. }\end{array}$ \\
\hline
\end{tabular}


de la composición química de sus aceites esenciales-, varios quimiotipos de pronto alivio (Lippia alba); en la Figura 5 aparece su distribución geográfica [50]. En Colombia, prevalecen quimiotipos "Carvona" y "Citral"; similarmente, en Brasil.

Para las plantas del orégano de monte (Lippia origanoides), con base en el análisis cromatográfico detallado de sus aceites esenciales, se encontraron tres quimiotipos, llamados así por sus componentes mayoritarios: "Timol", "Carvacrol" y "Felandreno". El Análisis de Componentes Principales (PCA), junto con la información detallada de la composición del aceite, constituyen una herramienta base para la clasificación de quimiotipos [51]. Mientras que los aceites esenciales de los quimiotipos de Lippia origanoides "Timol" y "Carvacrol" presentan una alta actividad antioxidante [24], el aceite esencial aislado del quimiotipo "Felandreno", no exhibe

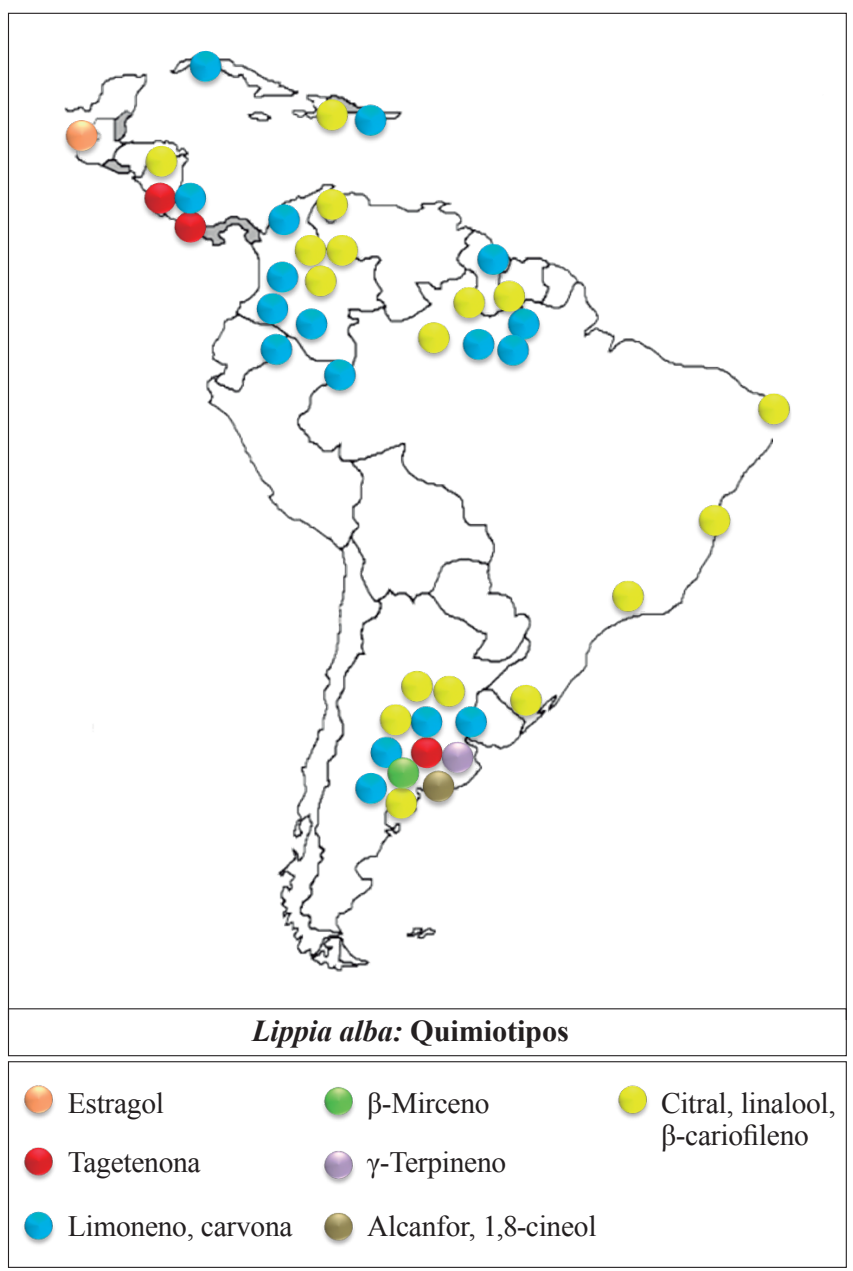

Figura 5. Distribución geográfica de quimiotipos de la especie vegetal Lippia alba. La asignación de quimiotipos está dada por el componente o componentes mayoritarios en el aceite esencial, según [50]. propiedades antioxidantes (atrapamiento de radicales libres). Para los extractos hidro-alcohólicos u obtenidos con fluido supercrítico ( $\left.\mathrm{SFE}-\mathrm{CO}_{2}\right)$, se presenta un cuadro totalmente diferente. El extracto de Lippia origanoides, quimiotipo "Felandreno", rico en flavonoides, particularmente, en pinocembrina, posee una alta actividad antioxidante, mientras para los extractos obtenidos de otros quimiotipos, esta actividad fue muy baja [52]. Es curioso anotar, que las plantas (flores, en particular) de Lippia origanoides, quimiotipo "Felandreno", se marchitan mucho más lento, que las de los otros dos quimiotipos, "Timol" y "Carvacrol". Ello se explica, posiblemente, por el contenido alto de flavonoides en las plantas del quimiotipo "Felandreno".

\section{Materiales y métodos}

\subsection{Material vegetal}

El material vegetal provino de colectas botánicas realizadas en diferentes localidades colombianas, bajo el permiso otorgado por el Ministerio de Ambiente (Resolución 0041 del 11 de enero de 2008) para el estudio con fines de investigación científica en diversidad biológica, con acceso a productos derivados con fines de investigación y bioprospección. La Tabla 3 presenta los resultados de la caracterización taxonómica realizada en el Instituto de Ciencias Naturales, el número con el que se registró la copia del material vegetal depositada en el Herbario Nacional Colombiano, las localidades y departamentos en donde se hizo la colecta y el rendimiento obtenido en la extracción del aceite esencial (AE).

\subsection{Materiales y reactivos}

Acetato de geranilo, alcanfor, allo-aromadendreno, ß-bourboneno, borneol, canfeno, trans- $\beta$-cariofileno, carvacrol, carvona, $p$-cimeno, 1,8-cineol, $\alpha$-copaeno, cis-dihidrocarvona, trans-dihidrocarvona, citronelal, $\beta$-elemeno, espatunelol, eugenol, $\alpha$-felandreno, $\beta$-felandreno, geranial, geraniol, germacreno D, $\alpha$-humuleno, limoneno, linalol, 6-metil-5hepten-2-ona, ß-mirceno, neral, nerol, 1-octen-3-ol, óxido de cariofileno, cis-óxido de limoneno, $\alpha$-pineno, $\beta$-pineno, piperitenona, piperitona, sabineno, $\alpha$-terpineno $\gamma$-terpineno, terpinoleno, timol, trans-nerolidol, y trans-óxido de limoneno, se obtuvieron con pureza de $98 \%$ o mayor, de Sigma-Aldrich (Steinheim, Alemania). Tetradecano, patrón para cromatografía, se obtuvo de Merck (Darmstadt, Alemania). Fluoresceína, ácido 2,2'-azinobis-(3-etilben-zotiazolina-ácido sulfónico) (ABTS), clorhidrato de 2,2'-azobis(2-amidinopropano) (AAPH), Trolox ${ }^{\circledR}$ (ácido 6-hidroxi-2,5,7,8-tetrametilcroman-2-carboxílico), persulfato de potasio, $\alpha$-tocoferol, y 2,6-bis(1,1-dimetiletil)-4-metilfenol (BHT), se obtuvieron de Merck (Darmstadt, Alemania). Metanol y etanol grado analítico fueron suministrados por Mallinckrodt Baker Inc. (Phillipsburgh, PA, EE.UU.). Se utilizó agua tipo I obtenida en un equipo Millipore (Millipore S. A., Molsheim, Francia). 
Tabla 3. Número de registro (Herbario Nacional Colombiano) y rendimiento de extracción del AE de plantas del género Lippia sp., recolectadas en diferentes regiones de Colombia.

\begin{tabular}{|c|c|c|c|c|}
\hline Especie & Localidad & Departamento & Número de registro & Rendimiento, $\% \mathrm{p} / \mathrm{p}$ \\
\hline L. alba (carvona) & Venadillo & Tolima & 520287 & 0,8 \\
\hline L. alba (citral) & Turbaco & Bolívar & 516929 & 0,9 \\
\hline L. alba (carvona + citral) & Saravena & Arauca & 512084 & 0,4 \\
\hline L. alba (mircenona) & Bucaramanga & Santander & 560257 & 0,2 \\
\hline L. origanoides (timol) & Pedregal & Nariño & 520285 & 3,1 \\
\hline L. origanoides (carvacrol) & Piedecuesta & Santander & 516290 & 4,4 \\
\hline L. origanoides (felandreno) & Los Santos & Santander & 516294 & 1,5 \\
\hline L. citriodora & Sucre & Santander & 555845 & 0,1 \\
\hline L. micromera & Manaure & Cesar & 516924 & 1,0 \\
\hline L. americana & Los Santos & Santander & 516287 & 0,5 \\
\hline L. graveolens & Bucaramanga & Santander & 555839 & 1,2 \\
\hline L. dulcis & Bucaramanga & Santander & 512079 & 0,2 \\
\hline
\end{tabular}

\subsection{Procedimiento de extracción}

La hidrodestilación asistida por la radiación de microondas empleó un horno microondas doméstico (LG, $800 \mathrm{~W}, 2,4$ $\mathrm{GHz}$ ) provisto de un orificio lateral a través del cual se acopló un condensador externo de vidrio con un balón de fondo redondo de $2 \mathrm{~L}$, que contenía el material vegetal (100 $-300 \mathrm{~g})$ y agua $(300-500 \mathrm{~mL})$, dentro del horno. El horno se operó por 3 sesiones consecutivas de 8 minutos con 30 $\mathrm{s}$ de descanso, lo que ocasionó la generación del vapor de agua, que junto con el aceite esencial liberado, se mantuvo en reflujo gracias al condensador. La inserción de una trampa Dean-Stark entre el condensador y el balón permitió la separación por densidad, del aceite esencial y el agua condensados.

\subsection{Análisis cromatográfico}

Los aceites esenciales de cada especie se analizaron en un cromatógrafo Agilent Technologies 6890 Plus (Palo Alto, CA, EE.UU.), acoplado a un detector selectivo de masas Agilent Technologies MSD 5973 y equipado con un puerto de inyección split/splitless $\left(250^{\circ} \mathrm{C}\right.$, relación split 1:30) y un inyector automático Agilent 7863. La separación de los componentes del aceite esencial se realizó en una columna capilar de sílice fundida DB-5MS ( $J \& W$ Scientific, Folsom, CA, EE.UU.), de $60 \mathrm{~m} \times 0.25 \mathrm{~mm}$, d.i. x $0.25 \mu \mathrm{m}, \mathrm{d}_{\mathrm{f}}$, con fase estacionaria de 5\%-fenil-poli(metilsiloxano), y otra de sílice fundida DB-WAX ( $J \& W$ Scientific, Folsom, CA, EE.UU.), con fase estacionaria entrecruzada e inmovilizada de poli(etilenglicol) de $60 \mathrm{~m} \times 0.25 \mathrm{~mm}$, d.i. $\times 0.25 \mu \mathrm{m}, \mathrm{d}_{\mathrm{f}}$. El gas de arrastre utilizado fue helio (He, 99.9995\%, Linde Colombia, S.A.), con flujo constante de $1 \mathrm{~mL} \mathrm{m^{-1 }}$. La programación de temperatura del horno fue $45^{\circ} \mathrm{C}(5 \mathrm{~min})$ a $4{ }^{\circ} \mathrm{C}$ min $^{-1}$ hasta $150{ }^{\circ} \mathrm{C}(2 \mathrm{~min})$ a $5{ }^{\circ} \mathrm{C} \mathrm{min}{ }^{-1}$ hasta $250{ }^{\circ} \mathrm{C}$
(5 min) y, finalmente, a $10{ }^{\circ} \mathrm{C} \mathrm{min}{ }^{-1}$ hasta $275{ }^{\circ} \mathrm{C}(15 \mathrm{~min})$. Los componentes de los aceites esenciales se identificaron por comparación de sus espectros de masas, obtenidos por GC-MS, e índices de retención (polar y apolar, calculados con base en la serie homóloga de $n$-alcanos $\mathrm{C}_{9}-\mathrm{C}_{25}$ (SigmaAldrich, St Louis, MI, EE.UU.), con los de bases de datos (Adams 2007, Wiley 138K, NIST 2002) y de patrones certificados, obtenidos bajo idénticas condiciones.

El análisis cuantitativo se efectuó utilizando un cromatógrafo Agilent Technologies 7890A (HP, Palo Alto, California, EE.UU.), equipado con un detector de ionización en llama (FID), con puerto de inyección split/splitless $\left(250^{\circ} \mathrm{C}\right.$, relación split 1:30), y sistema de inyección automático Agilent 7683 $B$. La separación de los componentes del aceite esencial se realizó en una columna con fase estacionaria apolar DB-5MS (J \& W Scientific, Folsom, CA, EE.UU.) de $60 \mathrm{~m} \times 0.25$ $\mathrm{mm}$, D.I. x $0.25 \mu \mathrm{m}, \mathrm{d}_{\mathrm{f}}$, con fase estacionaria de $5 \%$-fenilpoli(metilsiloxano). El gas de arrastre utilizado fue helio (He, 99.9995\%, Linde Colombia, S.A.), con flujo constante de $1 \mathrm{~mL} \mathrm{~min}^{-1}$. La programación de temperatura del horno fue $45^{\circ} \mathrm{C}(5 \mathrm{~min})$ a $4{ }^{\circ} \mathrm{C} \mathrm{min}{ }^{-1}$ hasta $150{ }^{\circ} \mathrm{C}(2 \mathrm{~min})$ a $5{ }^{\circ} \mathrm{C}$ $\mathrm{min}^{-1}$ hasta $250^{\circ} \mathrm{C}(5 \mathrm{~min}) \mathrm{y}$, finalmente, a $10^{\circ} \mathrm{C} \mathrm{min}^{-1}$ hasta $275^{\circ} \mathrm{C}(15 \mathrm{~min})$.

\subsection{Capacidad antioxidante}

La determinación de la capacidad antioxidante de los AE y las sustancias de referencia se efectuó en un lector de microplacas Turner Biosystems Inc., Modulus ${ }^{T M}$ II Microplate Multimode Reader (Sunnyvale, CA, EE. UU.), con microplacas de poli(estireno) de 96 pozos y equipado con módulos de absorbancia (UV-Vis) y fluorescencia. El llenado de las microplacas se realizó con una Transferpette ${ }^{\circledR}-8 /-12$ Electronic (Brand, Alemania). Las mediciones se realizaron 
siguiendo dos metodologías, i.e., la capacidad antioxidante total equivalente al Trolox ${ }^{\circledR}$ (TEAC) y la capacidad de absorción de los radicales de oxígeno (ORAC). Todas las mediciones se realizaron por triplicado y los resultados se expresaron como la media \pm desviación estándar.

\subsubsection{Método TEAC}

Para evaluar la actividad antioxidante de los AE y patrones según la metodología TEAC se siguió el procedimiento reportado por Re et. al. [53] con algunas modificaciones realizadas por Arias [54]. Las lecturas de absorbancia se realizaron a $750 \mathrm{~nm}$.

Se preparó una solución del catión-radical $\mathrm{ABTS}^{+\cdot}$, a partir de las soluciones de ABTS $(7 \mathrm{mM})$ y persulfato de potasio $(2.45 \mathrm{mM})$. La mezcla se homogeneizó en ultrasonidos por 30 min y se guardó por $24 \mathrm{~h} \mathrm{a} 4^{\circ} \mathrm{C}$, en ausencia de luz. A partir de esta solución, diariamente, se prepararon soluciones de trabajo con absorbancias $0.710 \pm 0.003$. Las muestras de AE $\mathrm{y}$ las sustancias de referencia, se diluyeron en etanol hasta que su adición a cierto volumen de la solución de trabajo de ABTS $^{+}$, resultara en una inhibición entre el 10 y $80 \%$ del blanco de absorbancia. La respuesta-concentración de las sustancias de referencia y AE, se calculó como porcentaje de la absorbancia del catión-radical $\mathrm{ABTS}^{+\cdot}$ sin inhibir (blanco de reacción), de acuerdo con la ecuación (1):

$$
\text { Inhibición de } \mathrm{A}_{\lambda: 750}(\%)=\frac{1-\mathrm{Af}}{\mathrm{Ao}} \times 100
$$

Donde:

Af: Absorbancia medida 30 min después de la adición del posible antioxidante.

Ao: Absorbancia del blanco de reacción (catión-radical sin inhibir).

Todos los ensayos se realizaron por triplicadoy se establecieron los valores de capacidad antioxidante equivalente al Trolox ${ }^{\circledR}$ ( $\mu \mathrm{mol}$ Trolox ${ }^{\circledR} / \mathrm{g}$ antioxidante evaluado).

\subsubsection{Método ORAC}

Esta metodología se implementó con base en el procedimiento reportado por Huang et al. [55] y las modificaciones de Arias [54], empleando el módulo de fluorescencia con filtros para longitudes de onda de $490 \mathrm{~nm}$ (excitación) y 510$570 \mathrm{~nm}$ (emisión).

Se utilizó una solución madre de fluoresceína (FL, 0,0838mM) en buffer fosfato $75 \mathrm{mM}(\mathrm{pH} \mathrm{7,4)} \mathrm{como} \mathrm{indicador} \mathrm{molecular.}$ A partir de esta se preparó, diariamente, una solución de trabajo de FL. Los radicales peroxilo se generaron a partir del AAPH $(153 \mathrm{mM})$. Una vez preparadas las soluciones se almacenaron en ausencia de luz a $4^{\circ} \mathrm{C}$. Posteriormente, se realizó la incubación de la muestra y la solución de FL a $37{ }^{\circ} \mathrm{C}$ por 20 minutos. Una vez finalizado el tiempo de incubación se adicionó la solución de AAPH con una pipeta multicanal Transferpette ${ }^{\circledR}$ en un tiempo máximo de 1 minuto.
La capacidad antirradicalaria de las muestras fue determinada con base en mediciones frecuentes de la disminución de la fluorescencia. Una vez finalizado el experimento, los datos de fluorescencia, en función del tiempo, se normalizaron con respecto al blanco de reacción (Fluorescencia inicial ${ }_{\text {Blanco }} /$ Fluorescencia inicial ${ }_{\text {Muestra }}$ ). Luego se calculó el área bajo la curva $(\mathrm{ABC})$, aplicando la ecuación (2):

$$
\mathrm{ABC}=1+\sum \mathrm{Fi} / \mathrm{Fo}
$$

Donde:

Fi : Fluorescencia en el tiempo i

Fo: Fluorescencia inicial ( 0 minutos)

El valor neto de $\mathrm{ABC}$ se obtuvo restando el $\mathrm{ABC}$ del blanco de reacción del $\mathrm{ABC}$ de cada una de las muestras analizadas. El Trolox ${ }^{\circledR}$ se utilizó como estándar de control en las mediciones. Los valores netos de $\mathrm{ABC}$ calculados se extrapolaron en una curva de calibración realizada con este estándar, y los resultados se expresaron en $\mu \mathrm{mol}$ Trolox ${ }^{\circledR} / \mathrm{g}$ antioxidante evaluado).

\section{Resultados y discusión}

3.1 Análisis composicional comparativo de los aceites esenciales de siete especies del género Lippia y sus quimiotipos

Los aceites esenciales se obtuvieron con rendimientos entre 0,2 y $4,4 \%$ (Tabla 3 ). Se destacan los rendimientos obtenidos con los quimiotipos de la especie L. origanoides $(1,5,3,1$ y $4,4 \%$ ), ya que el valor que se obtiene con muchas especies está alrededor de $0,5 \%$. Estos valores altos favorecen la viabilidad económica de un posible aprovechamiento a escala industrial.

El análisis por cromatografía de gases acoplada a espectrometría de masas permitió la identificación de 102 sustancias diferentes en los 12 aceites esenciales de las especies vegetales bajo estudio (Tabla 4). Los componentes identificados representaron entre el 83,9\% (L. americana) y el $99,1 \%$ (L. micromera) del área cromatográfica total. Los compuestos oxigenados fueron los más abundantes y constituyeron la familia de compuestos predominante en 9 de los 12 aceites esenciales. Los monoterpenos oxigenados representaron entre el 49,7 y el 71,6\% de la composición de los aceites de L. citriodora y de los 4 quimiotipos de $L$. alba . Similarmente, compuestos oxigenados (fenilpropanoides) constituyeron entre el 47,7 y el 78,1\% de la composición de los aceites de L. micromera, L. graveolens y los quimiotipos de $L$. origanoides ricos en timol y en carvacrol (Tabla 4). Los hidrocarburos sesquiterpénicos sumaron cantidades relativas de 52,3 y $54 \%$ en los aceites de L. americana y L. dulcis, respectivamente. Finalmente, los hidrocarburos monoterpénicos fueron la familia dominante $(45,7 \%)$ en la composición del aceite esencial del quimiotipo de $L$. origanoides rico en felandreno. 
Tabla 4. Composición química de los aceites esenciales del género Lippia.

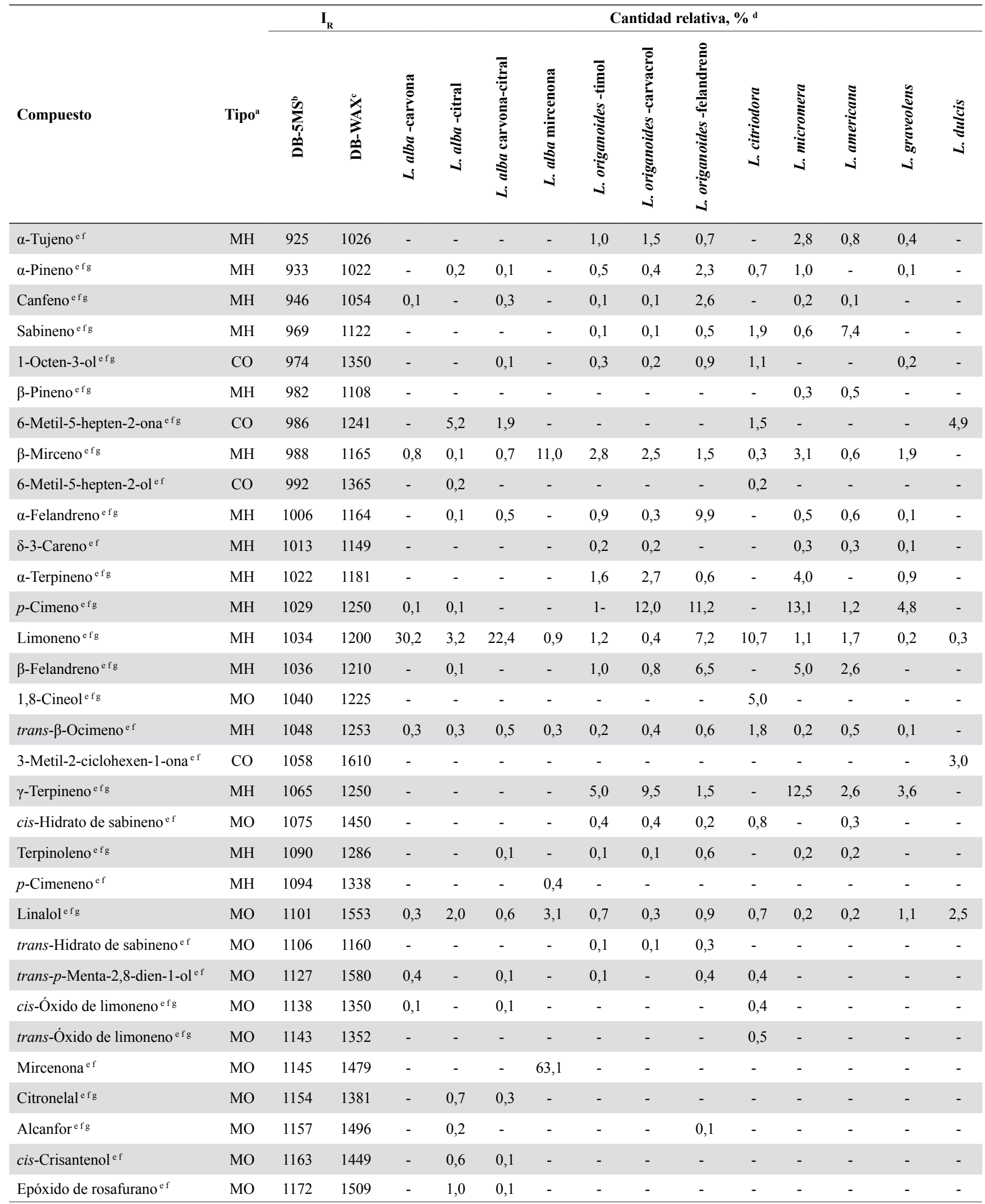




\begin{tabular}{|c|c|c|c|c|c|c|c|c|c|c|c|c|c|c|c|}
\hline Borneol $^{\text {efg }}$ & MO & 1182 & 1709 & 0,3 & 0,8 & 0,6 & - & - & 0,1 & 3,1 & - & 0,8 & 0,1 & - & - \\
\hline Umbelulona ${ }^{\text {ef }}$ & MO & 1188 & 1652 & - & - & - & - & - & - & - & - & 0,8 & - & - & - \\
\hline Terpinen-4-ol ${ }^{\text {ef }}$ & MO & 1189 & 1577 & - & - & - & - & 0,7 & 1,1 & 1,3 & - & 0,4 & 0,3 & 0,6 & - \\
\hline$\alpha$-Terpineol ${ }^{\text {ef }}$ & $\mathrm{MO}$ & 1203 & 1705 & - & - & - & & 0,1 & - & 1,1 & 1,7 & 0,1 & - & - & \\
\hline cis-Dihidrocarvona ${ }^{\mathrm{efg}}$ & MO & 1204 & 1517 & 0,3 & - & 0,4 & - & - & - & - & 1,0 & - & - & - & - \\
\hline trans-Dihidrocarvona $\mathrm{e}^{\mathrm{fg} g}$ & MO & 1212 & 1537 & 0,5 & - & 0,3 & - & - & - & - & - & - & - & - & - \\
\hline Citronelol $^{\text {efg }}$ & MO & 1225 & 1570 & - & - & - & - & - & - & - & - & - & - & - & 0,6 \\
\hline cis-Ocimenona $^{\mathrm{ef}}$ & $\mathrm{MO}$ & 1230 & 1568 & - & - & - & 4,8 & - & - & - & - & - & - & - & - \\
\hline Nerol $^{\text {efg }}$ & MO & 1230 & 1708 & - & 3,0 & 0,7 & - & - & - & - & 2,0 & - & - & - & - \\
\hline Metil timil éter ${ }^{\mathrm{ef}}$ & $\mathrm{CO}$ & 1232 & 1599 & - & - & - & - & 1,9 & 1,0 & - & - & 14,9 & - & 0,6 & - \\
\hline trans-Ocimenona ${ }^{\text {ef }}$ & MO & 1238 & 1578 & - & - & - & 0,6 & - & - & - & - & - & - & - & - \\
\hline Neral ${ }^{\text {efg }}$ & $\mathrm{MO}$ & 1247 & 1589 & - & 21,0 & 10,4 & - & - & - & - & 15,6 & - & - & - & 0,5 \\
\hline Carvona $\mathrm{e}^{\mathrm{fg} g}$ & MO & 1253 & 1732 & 50,3 & - & 25,3 & - & - & - & - & - & - & 0,7 & - & 1,2 \\
\hline Geraniol efg $^{\text {fg }}$ & MO & 1254 & 1755 & - & 6,0 & - & - & - & - & - & 2,7 & - & - & 0,3 & - \\
\hline Piperitona $\mathrm{e}^{\mathrm{efg}}$ & MO & 1264 & 1641 & 3,1 & 0,1 & 1,1 & - & - & - & - & - & - & - & - & - \\
\hline Geranial efg & MO & 1277 & 1741 & - & 27,0 & 10,4 & - & - & - & - & 18,9 & 0,5 & - & - & 0,8 \\
\hline Timol $^{\text {efg }}$ & $\mathrm{CO}$ & 1290 & 2193 & - & - & 0,1 & - & 54,5 & 9,9 & 0,5 & - & 29,1 & - & 64,6 & - \\
\hline Carvacrol $^{\text {efg }}$ & $\mathrm{CO}$ & 1304 & 2172 & - & - & - & - & 1,7 & 46,2 & - & - & 1,0 & 0,2 & 12,2 & - \\
\hline$\delta$-Elemeno ${ }^{\text {ef }}$ & $\mathrm{SH}$ & 1345 & 1481 & - & - & - & - & - & - & - & - & - & 0,7 & - & 0,8 \\
\hline Piperitenona $\mathrm{e}^{\mathrm{f} g}$ & MO & 1350 & 1842 & 6,1 & - & 2,2 & - & - & - & - & - & - & - & - & - \\
\hline Acetato de timilo ef & $\mathrm{CO}$ & 1350 & 1858 & - & - & - & - & 4,8 & 0,1 & - & - & 2,7 & - & - & - \\
\hline Eugenol ${ }^{\text {f } g}$ & $\mathrm{CO}$ & 1358 & 2080 & - & 0,3 & 0,2 & - & 0,1 & 0,3 & 0,6 & 0,3 & - & - & 0,5 & - \\
\hline Acetato de geranilo ${ }^{\text {efg }}$ & $\mathrm{CO}$ & 1379 & 1662 & - & 0,6 & 0,4 & - & - & - & - & 1,3 & - & - & - & - \\
\hline$\alpha$-Copaeno efg & $\mathrm{SH}$ & 1387 & 1450 & - & - & 0,1 & - & 0,1 & - & 0,9 & 0,5 & - & 0,8 & - & 8,3 \\
\hline$\beta$-Cubebeno ${ }^{\text {f }}$ & $\mathrm{SH}$ & 1394 & 1466 & - & - & - & - & - & - & 0,1 & - & - & 2,2 & - & - \\
\hline$\beta$-Bourboneno ${ }^{\text {efg }}$ & $\mathrm{SH}$ & 1396 & 1517 & 1,0 & - & - & - & - & - & 0,2 & 0,8 & - & 1,3 & - & 1,2 \\
\hline$\beta$-Elemeno efg & $\mathrm{SH}$ & 1398 & 1496 & - & 0,8 & 1,7 & 1,3 & - & - & 0,3 & - & - & - & - & - \\
\hline trans- $\beta$-Cariofileno e fg & $\mathrm{SH}$ & 1420 & 1597 & - & 5,4 & 2,4 & - & 2,4 & 2,0 & 11,3 & 2,3 & 1,9 & 12,2 & 2,9 & 9,9 \\
\hline trans $-\beta$-Farneseno $^{\text {ef }}$ & $\mathrm{SH}$ & 1442 & 1668 & - & - & - & 0,8 & - & - & - & - & - & - & - & 2,9 \\
\hline$\beta$-Gurjuneno ef & $\mathrm{SH}$ & 1444 & 1447 & 0,1 & - & 0,2 & - & - & - & 1,0 & - & - & - & - & - \\
\hline$\beta$-Copaeno ${ }^{\text {f }}$ & $\mathrm{SH}$ & 1446 & 1607 & - & - & - & - & - & - & - & - & - & 0,6 & - & - \\
\hline$\alpha$-Guaieno ${ }^{\text {ef }}$ & $\mathrm{SH}$ & 1448 & 1498 & - & 1,4 & 1,3 & - & - & - & - & - & - & - & - & - \\
\hline Sesquisabineno ${ }^{\mathrm{ef}}$ & $\mathrm{SH}$ & 1459 & 1647 & - & - & - & - & - & - & - & - & - & - & - & 2,7 \\
\hline allo-Aromadendreno ${ }^{\mathrm{e} f \mathrm{~g}}$ & $\mathrm{SH}$ & 1460 & 1700 & - & - & - & - & - & - & - & - & - & - & 0,1 & 2,1 \\
\hline$\gamma$-Muuroleno ef & $\mathrm{SH}$ & 1464 & 1709 & - & - & - & - & - & - & - & - & - & 0,5 & - & 1,2 \\
\hline$\alpha$-Humuleno ${ }^{\text {efg }}$ & $\mathrm{SH}$ & 1452 & 1674 & - & 1,6 & 0,5 & - & 1,5 & 1,5 & 6,0 & - & 0,2 & 2,6 & 1,5 & 0,7 \\
\hline$\gamma$-Gurjuneno ${ }^{\text {ef }}$ & $\mathrm{SH}$ & 1476 & 2210 & 0,1 & 0,1 & 0,3 & - & 0,1 & 0,1 & 1,0 & 0,5 & - & 1,2 & - & - \\
\hline$\gamma$-Muuroleno ${ }^{\text {ef }}$ & $\mathrm{SH}$ & 1486 & 1697 & - & - & 0,1 & - & 0,2 & 0,1 & 1,3 & - & 0,2 & - & - & - \\
\hline $\operatorname{ar}$-Curcumeno ${ }^{\mathrm{ef}}$ & $\mathrm{SH}$ & 1488 & 1775 & - & - & - & - & - & - & 0,2 & 3,9 & - & - & - & - \\
\hline trans-Muurola-4(15), 5-dieno ${ }^{\text {ef }}$ & $\mathrm{SH}$ & 1493 & 1609 & - & - & - & - & - & - & - & - & - & - & - & 4,3 \\
\hline
\end{tabular}




\begin{tabular}{|c|c|c|c|c|c|c|c|c|c|c|c|c|c|c|c|}
\hline Biciclosesquifelandreno ${ }^{\text {ef }}$ & $\mathrm{SH}$ & 1494 & 1624 & 3,5 & 1,5 & 8,0 & - & - & - & - & - & - & - & - & - \\
\hline Germacreno $D^{\text {efg }}$ & $\mathrm{SH}$ & 1495 & 1719 & - & - & 0,1 & 4,5 & 0,1 & 0,1 & 0,7 & 1,4 & 0,4 & 16,3 & 0,3 & - \\
\hline$\alpha$-Zingibereno ${ }^{\text {ef }}$ & SH & 1501 & 1719 & - & - & - & - & - & - & 0,3 & 0,3 & - & - & - & - \\
\hline Isobutanoato de geranilo $^{\mathrm{ef}}$ & $\mathrm{CO}$ & 1508 & 1633 & - & 0,6 & - & - & - & - & - & - & - & - & - & - \\
\hline$\alpha$-Muuroleno ef & $\mathrm{SH}$ & 1508 & 1736 & - & - & - & - & - & - & 0,7 & - & 0,2 & - & - & - \\
\hline$\beta$-Selineno ${ }^{\text {ef }}$ & $\mathrm{SH}$ & 1509 & 1744 & - & - & - & - & - & - & 1,2 & - & - & 1,7 & - & - \\
\hline Biciclogermacreno ${ }^{\text {f }}$ & SH & 1510 & 1750 & 0,2 & - & 0,7 & - & 0,1 & 0,1 & - & 1,3 & - & 5,6 & - & 8,0 \\
\hline$\alpha$-Bulneseno ${ }^{\text {ef }}$ & $\mathrm{SH}$ & 1515 & 1627 & - & 0,8 & 0,8 & - & - & - & - & - & - & - & - & - \\
\hline$\beta$-Bisaboleno ${ }^{\text {ef }}$ & $\mathrm{SH}$ & 1515 & 1745 & - & - & - & - & 0,6 & - & - & - & - & - & 0,1 & 3,0 \\
\hline$\gamma$-Cadineno ${ }^{\text {ef }}$ & $\mathrm{SH}$ & 1526 & 1789 & - & 0,3 & - & - & 0,1 & - & 0,8 & - & - & 0,5 & - & - \\
\hline Cubebol $^{\text {ef }}$ & SO & 1529 & 1855 & 0,3 & 0,2 & 0,4 & - & - & - & - & 1,0 & - & - & - & - \\
\hline$\delta$-Cadineno ${ }^{\text {ef }}$ & $\mathrm{SH}$ & 1530 & 1783 & - & - & - & - & 0,1 & - & 1,6 & - & - & 1,5 & 0,4 & 8,9 \\
\hline trans-Calameneno $^{\mathrm{ef}}$ & SH & 1534 & 1844 & - & - & - & - & - & - & 1,1 & - & 0,3 & - & - & - \\
\hline cis-Calameneno $^{\text {ef }}$ & SH & 1536 & 1845 & - & - & - & - & - & - & 0,4 & - & - & 0,4 & - & - \\
\hline trans-Cadino-1,4-dieno ef & SH & 1547 & 1783 & - & - & - & - & - & - & - & - & - & 0,6 & - & - \\
\hline$\alpha$-Cadineno ${ }^{\text {ef }}$ & SH & 1551 & 1799 & - & - & - & - & - & - & - & - & - & 0,6 & - & - \\
\hline trans-Nerolidol e fg & SO & 1566 & 1946 & - & 0,1 & - & - & - & - & 0,2 & 1,7 & - & - & - & 2,1 \\
\hline Germacreno $\mathrm{B}^{\mathrm{ef}}$ & $\mathrm{SH}$ & 1581 & 1840 & - & - & - & - & - & - & - & - & - & 3,0 & - & - \\
\hline Germacren-4-ol ${ }^{\text {f }}$ & SO & 1593 & 2123 & - & 0,1 & 0,2 & - & - & - & - & - & - & 2,8 & - & - \\
\hline Espatunelol ${ }^{\text {efg }}$ & SO & 1595 & 2043 & - & - & 0,1 & - & - & - & - & 4,7 & - & - & - & 4,5 \\
\hline Óxido de cariofileno $^{\text {e fg }}$ & SO & 1584 & 2005 & - & 2,2 & 0,4 & - & 0,4 & - & 2,2 & 5,0 & 0,5 & 2,0 & 0,1 & 3,4 \\
\hline$c i s$-Bisabolol ${ }^{\text {ef }}$ & SO & 1617 & 1626 & - & 1,0 & - & - & - & - & - & - & - & - & - & - \\
\hline Epóxido de humuleno II $^{\mathrm{ef}}$ & SO & 1629 & 1964 & - & 0,5 & - & - & - & - & - & - & - & - & 0,1 & - \\
\hline Cubenol $^{\mathrm{ef}}$ & SO & 1636 & 2057 & - & - & - & - & - & - & 0,2 & 0,4 & - & 3,6 & - & - \\
\hline$\gamma$-Eudesmol ${ }^{\text {f }}$ & SO & 1647 & 2184 & - & - & - & - & - & - & 0,6 & - & - & - & - & - \\
\hline trans-Asarona $^{\text {ef }}$ & $\mathrm{SO}$ & 1648 & 2314 & - & 1,0 & - & - & - & - & - & - & - & - & - & - \\
\hline$\alpha$-Muurolol ${ }^{\text {ef }}$ & $\mathrm{SO}$ & 1658 & 1660 & - & - & - & - & - & - & 0,3 & - & - & 0.4 & - & - \\
\hline$\alpha$-Cadinol ${ }^{\text {f }}$ & SO & 1672 & 2219 & - & - & - & - & - & - & 0,3 & - & - & 1,9 & - & - \\
\hline$\alpha$-Eudesmol ${ }^{\text {e f }}$ & SO & 1674 & 2116 & - & - & - & - & - & - & 1,4 & - & - & - & - & - \\
\hline$\alpha$-Bisabolol ${ }^{\mathrm{ef}}$ & SO & 1690 & 2229 & - & - & - & - & - & - & - & - & - & - & - & 9.1 \\
\hline \multicolumn{4}{|c|}{ Hidrocarburos monoterpénicos (MH) } & 31.5 & 4.1 & 24.6 & 12.6 & 14,7 & 31,0 & 45,7 & 15,4 & 44,9 & 19,1 & 12,2 & 0,3 \\
\hline \multicolumn{4}{|c|}{ Monoterpenos oxigenados (MO) } & 61.4 & 62.4 & 52.7 & 71.6 & 2,1 & 2,0 & 7,4 & 49,7 & 2,8 & 1,6 & 2,0 & 5,6 \\
\hline \multicolumn{4}{|c|}{ Compuestos oxigenados (CO) } & - & 6.9 & 2.7 & 0.0 & 63,3 & 57,7 & 2,0 & 4,4 & 47,7 & 0,2 & 78,1 & 7,9 \\
\hline \multicolumn{4}{|c|}{ Hidrocarburos sesquiterpénicos (SH) } & 4.9 & 11.9 & 16.2 & 6.6 & 5,3 & 3,9 & 29,1 & 11,0 & 3,2 & 52,3 & 5,3 & 54,0 \\
\hline \multicolumn{4}{|c|}{ Sesquiterpenos oxigenados (SO) } & 0.3 & 5.1 & 1.1 & - & 0,4 & - & 5,2 & 12,8 & 0,5 & 10,7 & 0,2 & 19,1 \\
\hline
\end{tabular}

a Tipo de compuesto: hidrocarburo monoterpénico $(\mathrm{MH})$, monoterpeno oxigenado (MO), compuesto oxigenados (CO), hidrocarburo sesquiterpénico (SH), sesquiterpeno oxigenado (SO)

b Índices de retención lineales determinados experimentalmente en columna DB-5MS (60 m)

c Índices de retención lineales determinados experimentalmente en columna DB-WAX (60 m)

d Promedio de tres extracciones (RSD 0,5-10\%)

e Identificación tentativa basada en los índices de retención lineales

f Identificación tentativa basada en los espectros de masas (EI, 70eV)

g Identificación basada en el uso de sustancias de referencia 
Al examinar las abundancias de compuestos individuales, se encontró que en 9 de los 12 aceites el constituyente mayoritario fue un compuesto oxigenado. El germacreno D $(16,3 \%$, L. americana) y el trans- $\beta$-cariofileno $(9,9 \%$, L. dulcis; $11,3 \%$, L. origanoides (felandreno)) fueron los constituyentes mayoritarios no-oxigenados. En 4 de los 12 aceites el componente mayoritario tuvo una abundancia superior al 50\% (carvona, 50,3\%, L. alba; timol, 54,5\%, L. origanoides; mircenona, $63,1 \%$, L. alba; timol, $64,6 \%$, L. graveolens). La mircenona se destaca porque solamente fue hallada en 1 aceite y en este fue el constituyente más abundante. El timol fue el compuesto encontrado como mayoritario con mayor frecuencia (L. micromera, 29,1\%, $L$. origanoides (timol) 54,5\% y L. graveolens $64,6 \%$ ).

El limoneno y el linalol, en cantidades relativas de 0,2 a 30,2 y 0,2 a $3,1 \%$, respectivamente, fueron las únicas sustancias comunes a todos los 12 aceites. Les siguieron en frecuencia de aparición, el $\beta$-mirceno $(0,1-11 \%)$ y el trans-ß-ocimeno $(0,1-1,8 \%)$, los cuales se detectaron en todos los aceites, a excepción del AE de L. dulcis. Los 4 quimiotipos de L. alba tuvieron solamente 4 sustancias en común (limoneno, linalol, ß-mirceno y trans-ß-ocimeno), presentes en cantidades relativas diferentes. Por otra parte, los 3 quimiotipos de L. origanoides tuvieron 25 sustancias en común, aunque las familias de compuestos predominantes fueron diferentes; $\mathrm{CO}$ para los quimiotipos ricos en timol y carvacrol y SH para el quimiotipo rico en felandrenos.

El análisis de componentes principales, basado en el coeficiente de correlación, aplicado a las composiciones presentadas en la Tabla 4, permitió representar de una manera compacta las similitudes y diferencias composicionales entre los 12 aceites esenciales. La Figura 6 contiene esta representación de las 12 composiciones halladas, en el espacio formado por los primeros $3 \mathrm{com}$ ponentes principales, que representan el $55,5 \%$ de la información. Las distancias entre las posiciones de los aceites esenciales representados son inversamente proporcionales a su similitud composicional. El quimiotipo de $L$. alba que posee tanto carvona como citral (a2, en la Figura 6), aparece efectivamente en un sitio intermedio entre los de los quimiotipos ricos solamente en citral (a1) o carvona (a3). Los quimiotipos de L. origanoides ricos en carvacrol (o1) o timol (o2) aparecen bastante próximos, aunque separados del quimiotipo rico en felandreno (o3), con el que poseen 25 constituyentes en común, pero de poca abundancia relativa (Tabla 5).

El factor 2 resultó ser el mejor discriminante según las familias de compuestos constituyentes. Los aceites cuya composición estuvo dominada por hidrocarburos mono- y sesquiterpénicos (L. origanoides-felandreno, L. dulcis, L. americana) tuvieron valores inferiores a -4 a lo largo de este eje. En la Figura
6 se aprecia que se podrían tomar como discriminantes los planos con Factor $2=-4$ y Factor $2=0,5$. Los aceites esenciales que tuvieron a los monoterpenos oxigenados como la principal familia de componentes (L. citriodora y los quimiotipos de $L$. alba) aparecen en esta representación con la segunda coordenada (Factor 2) superior a 0,5. O sea, a un solo lado del plano discriminante con Factor $2=0,5$. Los demás aceites esenciales, con compuestos mayoritarios oxigenados (fenilpropanoides) están ubicados en la Figura 6 entre los 2 planos discriminantes (Factor 2 entre -4 y $0,5)$. Así, la representación que se obtiene con el análisis de componentes principales facilita el establecimiento de similitudes y diferencias composicionales que no son inmediatamente aparentes al leer la Tabla 4. Estos resultados pueden aplicarse a la comparación composicional con aceites esenciales de otras especies $u$ otros quimiotipos.

A menudo, no es suficiente cuantificar los componentes del aceite esencial e identificarlos, es importante conocer también qué tipo de isómero óptico está presente. Por ejemplo, la carvona se encuentra en varias especies vegetales. El isómero óptico con la configuración (S)-(+)-carvona se halla en los aceites esenciales aislados de alcaravea (Carum carvi ) (50$70 \%$ ) y eneldo (Anethum graveolens) (40-60\%); mientras que el isómero con la configuración (R)-(-), está en el aceite

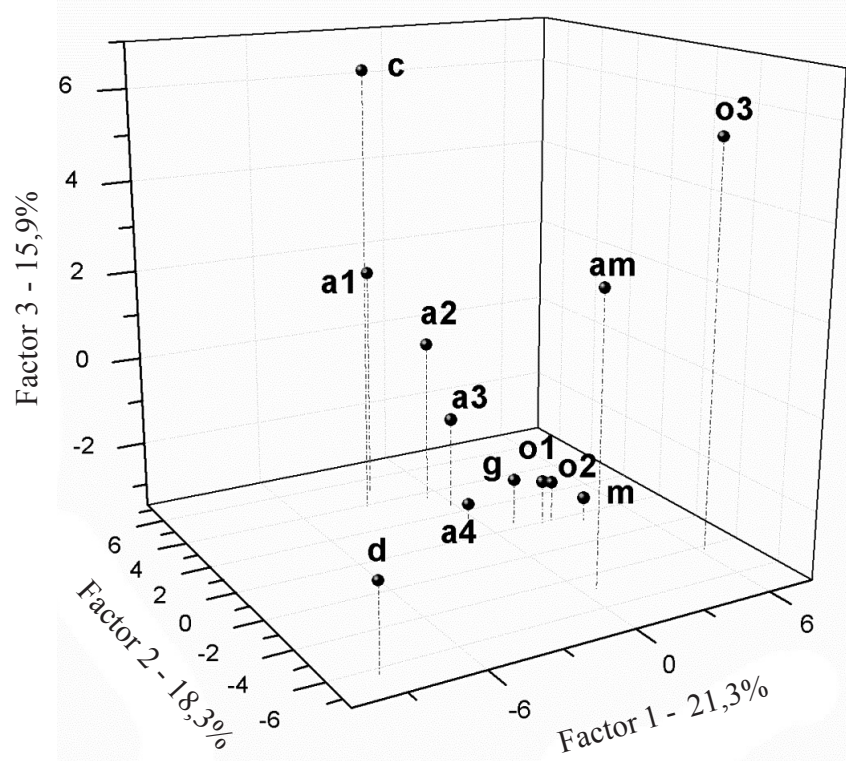

Figura 6. Representación de los aceites esenciales bajo estudio, en el sistema de coordenadas formado por los primeros 3 componentes principales, que representan el 55,5\% de la varianza total delos datos de composición. c, AE de L. citriodora; a1, AE de L. alba (citral); a2, AE de L. alba (carvona-citral); a3, AE de L. alba (carvona); a4, AE de L. alba (mircenona); g, AE de L. graveolens; o1, $\mathrm{AE}$ de L. origanoides (carvacrol); $\mathbf{0 2}, \mathrm{AE}$ de L. origanoides (timol); m, AE de $L$. micromera; am, AE de L. americana; $\mathbf{0 3}, \mathrm{AE}$ de $L$. origanoides (felandreno) y d, AE de L. dulcis. 
destilado de Mentha spicata (60-70\%). Los dos isómeros ópticos de la carvona son organolépticamente distintos; además, el isómero (S)-(+)-carvona es mucho más caro que su enantiómero. Uno de los quimiotipos de Lippia alba, que crece en Colombia, es rico en carvona. Para establecer su configuración, es decir, cuál enantiómero es, hay que hacer su análisis en una columna quiral, usando la configuración cromatográfica bidimensional (2D-GC), donde la primera columna, es "normal", y la segunda, unida a ella a través de un conmutador-sistema de microfluidos Deans switch, debe ser quiral. En la Figura 7 aparecen los cromatogramas de (A) la mezcla de (S)-(+)-carvona y (R)-(-)-carvona; (B) (S)$(+)$-carvona y $(\mathbf{C})$ la carvona presente en el aceite esencial de Lippia alba, que resulta tener la misma configuración, (4S)$(+)$-carvona, que la de alcaravea (Carum carvi) y eneldo (Anethum graveolens). Es un dato interesante, ya que (S)(+)-carvona es un enantiómero costoso, y la planta Lippia alba puede ser fuente para su aislamiento.

Un análisis similar por cromatografía bidimensional (2DGC) se llevó a cabo para determinar el tipo de isómero óptico del limoneno, presente en los aceites esenciales de L. alba, L. origanoides y L. citriodora. Este monoterpeno en L.alba y L. origanoides tiene la configuración de R-(+)limoneno, pero en $L$. citriodora, es su antípoda, i.e., S-()-limoneno. La determinación de la configuración óptica de componentes principales en los aceites esenciales es importante para su denominación de origen y el análisis de posibles falsificaciones.

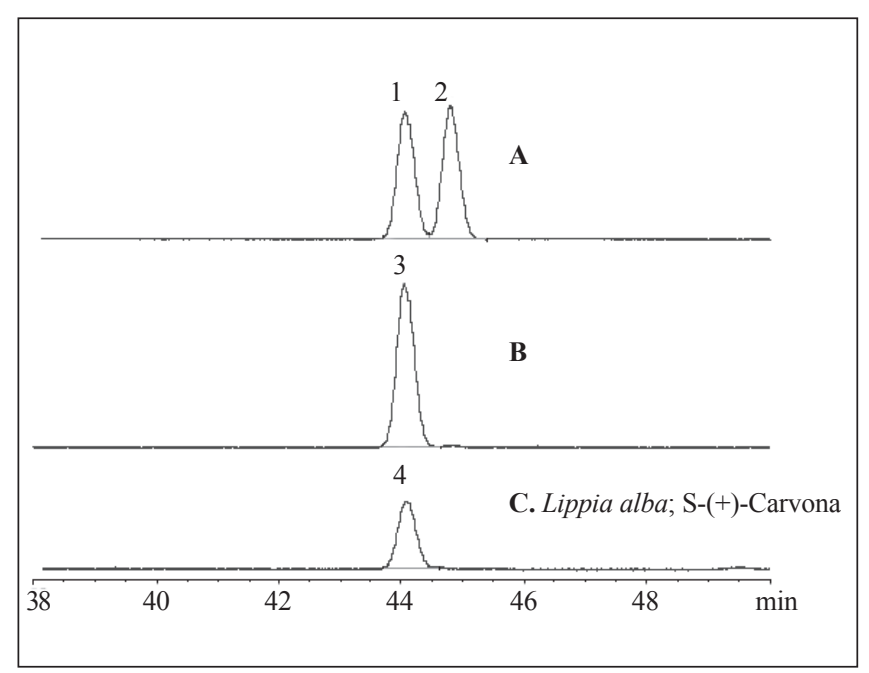

Figura 7. Perfiles cromatográficos de: A. Mezcla de patrones certificados de S-(+)-y R-(-)-carvona; B. Patrón certificado de S-(+)carvona; C. Aceite esencial de Lippia alba. En la configuración 2D-GC, la columna capilar quiral fue GAMMA DEX ${ }^{\mathrm{TM}} 120$ (30 $\mathrm{m} \times 0,25 \mathrm{~mm} \times 0,25 \mu \mathrm{m})$, acoplada a un detector de ionización en llama (FID).

\subsection{Estudio de la actividad antioxidante}

La Tabla 5 presenta los resultados de la evaluación de la capacidad antioxidante de los aceites esenciales y algunas sustancias individuales, por los métodos ORAC y ABTS. Los aceites esenciales son mezclas complejas de sustancias con diferentes funciones químicas que pueden exhibir reactividades variadas frente a especies radicales. La competencia entre las diferentes cinéticas de reacción, modulada por las cantidades relativas de los reactivos, da como resultado la capacidad antioxidante que se determina en el ensayo. Otro aspecto a considerar es la naturaleza del ensayo. Los métodos ORAC y ABTS exploran mecanismos de atrapamiento o aniquilamiento de radicales en los cuales el paso fundamental es la transferencia protónica o la transferencia electrónica, respectivamente [56].

Los resultados de la Tabla 5 muestran que los aceites esenciales ricos en fenilpropanoides (carvacrol, timol) mostraron un desempeño superior a los demás aceites, según los 2 métodos de evaluación empleados. La capacidad antioxidante ORAC de estos aceites fue 5 o más veces superior a la de sustancias utilizadas comúnmente en productos comerciales, tales como el BHT y el $\alpha$-tocoferol. Esta superioridad se mantuvo, aunque en menor proporción, en los resultados de capacidad antioxidante TEAC. Los valores ORAC y TEAC obtenidos para la evaluación del carvacrol y el timol individualmente, fueron cercanos a los registrados para los aceites que los contienen como componentes mayoritarios. Por esto, se puede afirmar que la capacidad antioxidante evaluada por los métodos ORAC y ABTS está determinada en estos aceites por la presencia de carvacrol y timol. En los demás casos no es tan clara esta relación entre la actividad antioxidante del aceite y la de sus componentes mayoritarios. Las evaluaciones de sustancias individuales mostraron que hidrocarburos insaturados no aromáticos (trans-ß-cariofileno, $\alpha$-felandreno, $\gamma$-terpineno) pueden secuestrar radicales bajo las condiciones del ensayo ORAC (transferencia protónica), pero son completamente inactivos bajo las condiciones del ensayo ABTS (transferencia electrónica). Estos resultados son consistentes con los observados para los aceites de $L$. origanoides (felandreno) y L. americana, cuyos componentes mayoritarios son hidrocarburos mono- y sesquiterpénicos no oxigenados. En los casos restantes, entre los componentes de mayor abundancia se encuentran sustancias oxigenadas (geranial, neral, carvona) que podrían estabilizar una especie radical resultante de la reacción con un radical. Tal como se observó en el caso de los aceites con componentes mayoritarios no oxigenados, en los aceites ricos en geranial y neral, o carvona, la capacidad antioxidante evaluada con el método ABTS fue alrededor de 10 veces menor que la que se evaluó con el ensayo ORAC, lo que indica que en estos compuestos carbonílicos el paso determinante de la actividad antioxidante es una transferencia protónica. Según 
Tabla 5. Valores de capacidad antioxidante $\left(\mu \mathrm{mol}\right.$ Trolox ${ }^{\circledR} / \mathrm{g}$ sustancia) obtenidos por los métodos ORAC y ABTS ${ }^{+}$para los $\mathrm{AE}$ de especies de Lippia y sustancias de referencia.

\begin{tabular}{|c|c|c|}
\hline \multirow[b]{2}{*}{ Muestra } & \multicolumn{2}{|c|}{ Métodos $(\operatorname{promedio} \pm \mathrm{s}, \mathrm{n}=3)$} \\
\hline & $\begin{array}{c}\text { ORAC } \\
\begin{array}{c}\mu \text { mol Trolox }^{\circledR} / g \\
\text { sustancia) }\end{array}\end{array}$ & $\begin{array}{c}\text { ABTS }^{+} \\
(\mu \text { mol Trolox } \\
\text { g sustancia) }\end{array}$ \\
\hline AE de $L$. graveolens & $3990 \pm 58$ & $5410 \pm 48$ \\
\hline AE de $L$. citriodora & $3630 \pm 40$ & $41 \pm 2$ \\
\hline AE de L. origanoides (carvacrol) & $3400 \pm 120$ & $5200 \pm 109$ \\
\hline AE de L. origanoides (timol) & $2840 \pm 72$ & $5090 \pm 42$ \\
\hline AE de L. micromera & $2050 \pm 78$ & $2750 \pm 80$ \\
\hline AE de L. alba (citral) & $2000 \pm 77$ & $24.8 \pm 0.4$ \\
\hline AE de L. origanoides (felandreno) & $1820 \pm 82$ & $310 \pm 2$ \\
\hline AE de $L$. alba (carvona) & $1340 \pm 54$ & $126.4 \pm 0.7$ \\
\hline AE de L. americana & $1200 \pm 27$ & $239 \pm 4$ \\
\hline Carvacrol & $3410 \pm 50$ & $4609 \pm 6$ \\
\hline Timol & $3000 \pm 103$ & $5700 \pm 125$ \\
\hline trans- $\beta$-cariofileno & $2800 \pm 109$ & N.D. \\
\hline$\gamma$-Terpineno & $1766 \pm 8$ & N.D. \\
\hline$\alpha$-Felandreno & $1040 \pm 18$ & $136 \pm 3$ \\
\hline$\alpha$-Tocoferol & $550 \pm 13$ & $2429 \pm 7$ \\
\hline BHT & $457 \pm 9$ & $4760 \pm 23$ \\
\hline 1,8-Cineol & $299 \pm 5$ & N.D. \\
\hline$p$-Cimeno & $219 \pm 2$ & N.D. \\
\hline
\end{tabular}

N.D. : No se observó disminución en la coloración

los resultados del ensayo ORAC (Tabla 5), todos los aceites esenciales evaluados tuvieron mayor capacidad antioxidante que las del BHT y del $\alpha$-tocoferol y por esto, se constituyen en candidatos para utilizarse como ingredientes de productos finales, en reemplazo de antioxidantes sintéticos.

\section{Conclusiones}

Las sustancias oxigenadas (monoterpenos o fenilpropanoides) fueron los constituyentes más frecuentemente encontrados en los aceites esenciales de las especies del género Lippia estudiados y desempeñan un papel determinante en la capacidad antioxidante exhibida por estos. Los quimiotipos de L. origanoides ricos en carvacrol o timol tuvieron un rendimiento de extracción relativamente alto $(>3 \%)$ y sus aceites esenciales mostraron una capacidad antioxidante (ORAC, TEAC) superior a la de antioxidantes bien establecidos, tales como el BHT y el $\alpha$-tocoferol, usados comúnmente en productos comerciales.

\section{Agradecimientos}

Los autores agradecen muchísimo al doctor José Luís Fernández, por la caracterización taxonómica de las plantas estudiadas; a la química Martha Cervantes, M. Sc., por las búsquedas cienciométricas; a los químicos Ginna Arias y Carlos Ruiz, por colaboración en el trabajo experimental. Sinceras gracias a Colciencias (contratos RC 432-2004, RC-245-2011), al Ministerio de Agricultura y Desarrollo Rural (contratos 2007V3400-129, 2008V3781-3740) y a la Gobernación de Santander (Dr. Horacio Serpa Uribe) - NEOMUNDO, por la financiación de nuestras investigaciones. La recolección del material vegetal se llevó a cabo con el permiso oficial de estudio con fines de investigación científica en diversidad biológica con acceso a productos derivados con fines de investigación y bioprospección, Resolución 0041 del 11 de enero de 2008, del Ministerio del Ambiente.

\section{Referencias bibliográficas}

[1]. Stashenko, E. E.; Martínez, C.R. Martínez, J.R.; Shibamoto, T. 1995. Catalytic transformation of anise Oil (Pimpinella anisum L.) over zeolite Y. Journal of High Resolution Chromatography, 18 (8): 501-503.

[2]. Puertas-Mejía, M.; Hillebrand, S.; Stashenko, E.; Winterhalter, P. 2002. In vitro radical scavenging activity of essential oils from colombian plants and fractions from oregano (Origanum vulgare L.) essential oil. Journal of Flavour and Fragrance, 17, 380-384.

[3]. Stashenko, E. E.; Acosta, R.; Martínez, J.R. 2000. Highresolution gas chromatographic analysis of the secondary metabolites obtained by subcritical fluid extraction from Colombian rue (Ruta graveolens L.). Journal of Biochemical and Biophysical Methods, 43, 379-390.

[4]. Stashenko, E. E.; Villa, H.; Combariza, M.Y. 1995. Study of compositional variation in Colombian rue oil (Ruta graveolens) by HRGC using different detection systems. Journal of Microcolumn Separation, 7 (2): 117-122.

[5]. Rodríguez, R.; Ruiz C.; Arias, G.; Castro, H.; Martínez, J.; Stashenko, E.E. 2012. Estudio comparativo de la composición de los aceites esenciales de cuatro especies del género Cymbopogon (Poaceae) cultivadas en Colombia. 11 (1): 440-448.

[6]. Combariza, M. Y.; Blanco, C.; Stashenko, E.E.; Shibamoto, T. 1994. Limonene concentration in lemon (Citrus volkameriana) peel oil as a function of ripeness. Journal of High Resolution Chromatography, 17 (9): 643-646.

[7]. Blanco, C.; Stashenko, E.; Combariza, M.Y.; Martínez, J.R. 1995. Comparative study of Colombian citrus oils by high resolution gas chromatography and gas chromatography-mass spectrometry Journal of Chromatography A, 697, 501-513.

[8]. Stashenko, E.E.; Martínez, R.; Pinzón, M.E.; Ramírez, J. 1996. Changes in chemical composition of catalytically hydrogenated orange oil (Citrus sinensis). Journal of Chromatography A, 752, 217-222.

[9]. Stashenko, E.E.; Martínez, J.R.; Macku, C.; Shibamoto, T. 1993. HRGC and GC-MS analysis of essential oil from 
Colombian ylang-ylang (Cananga odorata hook fil. et Thomson, forma genuina). Journal of High Resolution Chromatography, 16 (7): 441-444.

[10]. Stashenko, E.E.; Torres, W.; Martínez, J.R. 1995. A study of compositional variation in the essential oil of ylang-ylang (Cananga odorata hook. Fil. Et thomson, forma genuina) during flower development. Journal of High Resolution Chromatography, 18 (2): 101-104.

[11]. Stashenko, E.E.; Quiroz N.; Martínez, J.R. 1996. HRGC/ FID/NPD and HRGC/MSD study of Colombian ylang-ylang (Cananga odorata) oils obtained by different extraction techniques. Journal of High Resolution Chromatography, 19 (6): 353-358.

[12]. García-Barriga, H.G. 1992. Flora medicinal de Colombia, Botánica médica. Tercer Mundo, Bogotá.

[13]. Correa, J.E.; Bernal, H.Y. 1989. Especies vegetales promisorias de los países del Convenio Andrés Bello. Editorial Guadalupe, Bogotá.

[14]. Gupta, M.P. (Ed.) 1995. 270 Plantas Medicinales Iberoamericanas. CYTED-SECAB, Presencia, Bogotá.

[15]. Stashenko, E.E. 2010. Aceites esenciales. Publicaciones UIS, Universidad Industrial de Santander, Bucaramanga, Colombia, $180 \mathrm{p}$.

[16]. Stashenko, E.E.; Martínez, J.R. (Editores), 2012. Plantas aromáticas y aceites esenciales: estudio y aplicaciones. Publicaciones UIS, Universidad Industrial de Santander, Bucaramanga, Colombia, $314 \mathrm{p}$.

[17]. Stashenko, E.E.; Wiame, H.; Dassy, S.; Martínez, J.R.; Shibamoto, T. 1995. Catalytic transformation of copaiba oil (Copaifera officinalis) over zeolite ZSM-5. Journal of High Resolution Chromatography, 18 (1): 54-58.

[18]. Stashenko, E.E.; Puertas, M.A.; Combariza, M.Y. 1996. Volatile secondary metabolites from Spilanthes americana obtained by simultaneous steam distillation - solvent extraction and supercritical fluid extraction. Journal of Chromatography A, 752, 223-232.

[19]. Stashenko, E.E.; Cervantes, M.; Combariza, Y.; Martínez, J.R. 1999. HRGC-FID-MSD analysis of the secondary metabolites obtained by different extraction methods from Lepechinia schiedeana, and evaluation of its antioxidant activity in vitro. Journal of High Resolution Chromatography, 22 (6): 343-349.

[20]. Stashenko, E.E.; Jaramillo, B. E.; Martínez, J.R. 2004. HRGC/FID/MSD analysis of volatile secondary metabolites from Lippia alba (Mill.) N.E. Brown grown in Colombia and evaluation of their in vitro antioxidant activity. Journal of Chromatography A, 1025, 99-103.

[21]. Stashenko, E.E.; Martínez, J.R.; Jaramillo, B.E. 2004. Analysis of volatile secondary metabolites from Colombian Xylopia aromatica (Lamarck) by different extraction and headspace methods and gas chromatography. Journal of Chromatography A, 1025, 105-113.
[22]. Quintero A.; González de Colmenares, N.; Stashenko, E.E.; 2004. Aceite esencial de las hojas de Hyptis umbrosa Salzm extraído por diferentes técnicas. Acta Científica Venezolana, 55, 181-187.

[23]. Güette-Fernández, J.; Olivero-Verbel, J.; O’Byrne-Hoyos, I.; Jaramillo, B.; Stashenko, E. 2008. Chemical composition and toxicity against Artemia franciscana of the essential oil of Callistemon speciosus (sims) DC., collected in Bogota (Colombia). Journal of Essential Oil Research, 20 (3): 272275.

[24]. Stashenko, E.E.; Ruiz, C.; Muñoz, A.; Castañeda, M.; Martínez, J. 2008. Composition and antioxidant activity of essential oils of Lippia origanoides H.B.K. grown in Colombia. Natural Product Communications, 3 (4): 563-566.

[25]. Stashenko, E.E.; Martínez, J.R. 2007. Sampling volatile compounds from natural products with headspace/solidphase micro-extraction. Journal of Biochemical and Biophysical Methods, 70 (2): 231-242.

[26]. Stashenko, E.E.; Martínez, J.R.; 2008. Sampling flower scent for chromatographic analysis. Journal of Separation Science, 31, 2022-2031.

[27]. Meneses, R.; Ocazionez, R.; Martínez, J.R.; Stashenko, E.E. 2009. Inhibitory effect of essential oils obtained from plants grown in Colombia on yellow fever virus replication in vitro. Annals of Clinical Microbiology and Antimicrobials, 8, 8-13.

[28]. Bueno, J.G.; Martínez, J.R.; Stashenko, E.E.; Ribón, W. 2009. Actividad antituberculosa de plantas colombianas. Biomédica, 29, 51-60.

[29]. Pascual, M.E.; Slowing, K.; Carretero, E.; Sánchez, D.; Villar, A. 2001. Lippia: traditional uses, chemistry and pharmacology: a review. Journal of Ethnopharmacology, 76, 73-99.

[30]. Oladimeji, F.A.; Orafidiya, L.O.; Okeke, I.N. 2004. Physical properties and antimicrobial activities of leaf essential oil of Lippia multiflora Moldenke. International Journal of Aromatheraphy, 14, 162-168.

[31]. Santiag G.M.P.; Lemos, T.L.G.; Pessoa, O.D.L.; Arriaga, A.M.C.; Matos, F.J.A.; Lima, M.A.S.; Santos, H.S.; Lima, M.C.L.; Barbosa F.G.; Luciano, J.H.S.; Silveira E.R.; Menezes, M.H.A. 2006. Larvicidal activity against Aedes aegypti L. (Diptera: Culicidae) of essential oils of Lippia species from Brazil. Natural Product Communications, 1, 573-576.

[32]. Hennebelle T.; Sahpaz, S.; Joseph, H.; Bailleul, F. 2008. Ethnopharmacology of Lippia alba. Journal of Ethnopharmacology, 116, 211-222.

[33]. Nerio, L.S.; Olivero-Verbel, J.; Stashenko, E.E. 2009. Repellent activity of essential oils from seven aromatic plants grown in Colombia against Sitophilus zeamais Motschulsky (Coleoptera). Journal of Stored Products Research, 45, 212-214. 
[34]. Dos Santos F.J.; Lopes J.A.; Cito, G.L.; de Oliveira E.H.; de Lima S.G.; de A.M. Reis, F. 2004. Composition and biological activity of essential oils from Lippia origanoides H.B.K. Journal of Essential Oil Research, 16, 504-506.

[35]. Oliveira, D.R.; Leitão G.G.; Bizzo H.R.; Lopes, D.; Alviano, D.S.; Alviano, C.S.; Leitão, S.G. 2007. Chemical and antimicrobial analysis of essential oil of Lippia origanoides H.B.K. Food Chemistry, 101, 236-240.

[36]. Rodríguez, D.A.; Sanabria, M.E. 2005. Efecto del extracto de tres plantas silvestres sobre la rizoctoniosis, la mancha sureña del maíz y los patógenos que las causan. Interciencia, 30 (12): 739-744.

[37]. Velasco, J.; Rojas, J.; Salazar, P.; Rodríguez, M.; Díaz, T.; Morales, A.; Rondón, M. 2007. Antibacterial activity of the essential oil of Lippia oreganoides against multiresistant bacterial strains of nosocomial origin. Natural Product Communications, 2 (1): $85-88$.

[38]. Brown, M.J.; Henderson, D.E.; Hunt, C. 2006. Comparison of antioxidant properties of supercritical fluid extracts of herbs and the confirmation of pinocembrin as a principle antioxidant in Mexican Oregano (Lippia graveolens). Electronic Journal of Environment, Agriculture, and Food, 5 (2): 1265-1277.

[39]. Huxley, A. 1992. The New Royal Horticultural Society Dictionary of Gardening. Macmillan Press, México.

[40]. Verdcourt, B. 1992. Flora of Tropical East Africa.Verbenaceae. CRC Press, Boca Raton, Florida, EE.UU.

[41]. Kovàts E. 1965. Gas chromatographic characterization of organic substances in the retention index system. Advances in Chromatography, 1, 229-232.

[42]. Davies, N.W. 1990. Chromatographic retention índices of monoterpenes and sesquiterpenes on methyl silicone and Carbowax 20M phases. Journal of Chromatography, 503, $1-24$.

[43]. Jennings, W.; Shibamoto, T. 1980. Quantitative analysis of flavor and fragrance volatiles by glass capillary gas chromatography. Academic Press, New York.

[44]. Joulain, D.; König, W.A. 1998. The atlas of spectral data of sesquiterpene hydrocarbons. E. B. Verlag, Hamburg.

[45]. Adams, P. 2007. Identification of essential oil components by gas chromatography/mass spectrometry. $4^{\text {th }}$ Ed., Allured Publishing, Carol Stream, IL, EE.UU.

[46]. Stashenko, E.E.; Martínez, J.R. 2010. Algunos aspectos prácticos para la identificación de analitos por cromatografía de gases acoplada a espectrometría de masas. Scientia Chromatographica, 2 (1): 29-47.

[47]. Stashenko, E.E.; Martínez, J.R. 2010. Separar, fragmentar e integrar: la rutina de un análisis por GC-MS. Patrones de fragmentación de moléculas orgánicas. Scientia Chromatographica, 2 (2): 23-46.

[48]. Stashenko, E.E.; Martínez, J.R. 2010. GC y GC-MS: configuración del equipo versus aplicaciones. Scientia Chromatographica, 2 (3): 25-51.

[49]. Stashenko, E.E.; Martínez, J.R. 2010. GC-MS: Más de un analizador de masas, ¿para qué? Scientia Chromatographica, 2 (4): 33-59.

[50]. Hennebelle, T.; Sahpaz, S.; Dermont, C.; Joseph, H.; Bailleul, F. 2006. The essential oil of Lippia alba: analysis of samples from French overseas departments and review of previous works. Chemistry and Biodiversity, 3, 1116-1125.

[51]. Stashenko, E.E.; Martínez, J.R.; Ruiz, C.; Arias, G.; Durán, C.; Salgar, W.; Cala, M. 2010 Lippia origanoides chemotype differentiation base on essential oil GC-MS and principal component analysis. Journal of Separation Science, 33, 93-103.

[52]. Stashenko, E.E.; Martínez, J.R.; Cala, M.; Durán, D.C. Caballero, D. 2013. Chromatographic and mass spectroscopic characterization of essential oils and extracts from Lippia (Verbenaceae) aromatic plants. Journal of Separation Science, 36, 192-202.

[53]. Re, R.; Pellegrini, N.; Proteggente, A.; Pannala, A.; Yang, M.; Rice-Evans, C. 1999. Antioxidant activity applying an improved ABTS radical cation decolorization assay. Free Radicals in Biology, 26, 1231-1235.

[54]. Arias, G. 2012. Estudio de la composición química y la actividad antioxidante de aceites esenciales y extractos aislados de Salvia officinalis, Rosmarinus officinalis, Thymus vulgaris y Lippia citriodora, cultivadas en el municipio de Sucre (Santander). Tesis de maestría, Escuela de Química, Universidad Industrial de Santander, 66-70.

[55]. Huang, D.; Ou, B.; Hampsch-Woodill, M.; Flanagan, J.; Prior, R. 2002. High-Throughput assay of oxygen radical absorbance capacity (ORAC) using a multichannel liquid handling system coupled with a microplate fluorescence reader in 96-well format. Journal of Agricultural and Food Chemistry, 50, 4438-2239.

[56]. Huang, D.; Ou, B.; Prior, R. 2005. The chemistry behind antioxidant capacity assays. Journal of Agricultural and Food Chemistry, 53, 1841-1856. 